\title{
Extended geometry and gauged maximal supergravity
}

\author{
G. Aldazabal, ${ }^{a, b}$ M. Graña, ${ }^{c}$ D. Marqués ${ }^{d}$ and J.A. Rosabal ${ }^{a, b}$ \\ ${ }^{a}$ Centro Atómico Bariloche, 8400 S.C. de Bariloche, Argentina \\ ${ }^{b}$ Instituto Balseiro (CNEA-UNC) and CONICET, 8400 S.C. de Bariloche, Argentina \\ ${ }^{c}$ Institut de Physique Théorique, CEA/ Saclay, 91191 Gif-sur-Yvette Cedex, France \\ ${ }^{d}$ Instituto de Astronomía y Física del Espacio (CONICET-UBA), \\ C.C. 67 - Suc. 28, 1428 Buenos Aires, Argentina \\ E-mail: aldazaba@cab.cnea.gov.a, mariana.grana@cea.fr, \\ diegomarques@iafe.uba.ar, rosabalj@ib.cnea.gov.ar
}

ABSTRACT: We consider generalized diffeomorphisms on an extended mega-space associated to the U-duality group of gauged maximal supergravity in four dimensions, $E_{7(7)}$. Through the bein for the extended metric we derive dynamical (field-dependent) fluxes taking values in the representations allowed by supersymmetry, and obtain their quadratic constraints from gauge consistency conditions. A covariant generalized Ricci tensor is introduced, defined in terms of a connection for the generalized diffeomorphisms. We show that for any torsionless and metric-compatible generalized connection, the Ricci scalar reproduces the scalar potential of gauged maximal supergravity. We comment on how these results extend to other groups and dimensions.

Keywords: Flux compactifications, Supersymmetry and Duality, Extended Supersymmetry, String Duality

ARXIV EPRINT: 1302.5419 


\section{Contents}

1 Introduction 1

2 Generalized diffeomorphisms and fluxes 3

$\begin{array}{lll}3 & \text { Consistency constraints } & \mathbf{7}\end{array}$

3.1 The section condition 8

$\begin{array}{lll}3.2 & \text { Twisted constraints } & 10\end{array}$

4 A geometry for the extended space $\quad 12$

$\begin{array}{lll}4.1 & \text { Generalized connections and torsion } & 12\end{array}$

$\begin{array}{lll}4.2 & \text { Generalized metric compatibility } & 14\end{array}$

5 Generalized Ricci tensor and the scalar potential of gauged maximal $\begin{array}{ll}\text { supergravity } & \mathbf{1 5}\end{array}$

$\begin{array}{lll}5.1 & \text { Generalized Ricci tensor and the section condition } & 15\end{array}$

$\begin{array}{lll}5.2 & \text { Generalized Ricci tensor and twisted conditions } & 16\end{array}$

$\begin{array}{ll}\text { 5.3 Generalized Ricci scalar and scalar potential of maximal supergravity } & 18\end{array}$

6 Summary and outlook $\quad 19$

A Useful $\boldsymbol{E}_{\boldsymbol{7}}$ identities $\quad \mathbf{2 1}$

A.1 $S U(8)$ subgroup $\quad 21$

A.2 $S L(2) \times O(6,6)$ subgroup $\quad 22$

B The Weitzenböck connection and the algebra 22

C Representations, projectors and generalized diffeomorphisms $\quad 23$

$\begin{array}{ll}\text { C.1 Representations and projectors } & 23\end{array}$

C.2 Generalized diffeomorphisms 24

$\begin{array}{ll}\text { D The scalar potential from extended geometry } & 27\end{array}$

\section{Introduction}

The classical symmetry group of the massless fields of string theory is larger than that of the metric, namely the group of diffeomorphisms, and includes purely stringy transformations such as T- or S-duality. The program to rewrite the theory in a covariant language under $O(d, d)$, the group that includes T-duality, goes under the name of Generalized Geometry [1, 2], or Double Field Theory (DFT) [3-6] (the latter based on previous constructions on 
Double Geometry [7-11]). In Generalized Geometry, the tangent space, where the vectors generating diffeomorphisms live, is enlarged to include the one-forms corresponding to gauge transformations of the B-field. Instead, in DFT, which aims at providing a field theory approach for strings, the space itself is doubled, and the extra half of the coordinates can be thought of as the duals of winding modes. Both approaches are related when a section condition is imposed which, effectively, un-doubles the double space. This condition is sufficient for consistency of DFT, but only a relaxed version of it is necessary for gauge consistency at the classical level $[12,13]$.

By including also S-duality in the game, i.e. by promoting the covariance to the full U-duality group, the tangent space or the double space gets enlarged to an extended (or exceptional) generalized tangent space or a mega-space (a mega-torus [14, 15], in the case of toroidal backgrounds). The enlarging is such that it accounts for the symmetries corresponding to RR fields as well as the NSNS, or equivalently combines the D-brane charges together with the momentum and winding charges of the string. The exceptional tangent space is the starting block of Exceptional Generalized Geometry [16, 17] (EGG), while we will call the extension of DFT to the U-duality groups, first discussed in [18-21], "Extended Field Theory" (EFT). As DFT, EFT can be restricted by a section condition [22, 23], that also constraints the fields to depend on a reduced number of physical coordinates, but more generally this constraint can also be relaxed. The U-duality symmetry groups are the exceptional groups $E_{d+1}$ of toroidal compactifications, where $d$ is the dimension of the compactification space in string theory (or $d+1$ in M-theory, or rather in 11-dimensional supergravity).

An appealing feature of the extended space is that the stringy symmetries (diffeomorphisms plus gauge transformations of all the gauge fields) look just like diffeomorphisms, and are encoded in a generalized Lie derivative [22, 23]-[28], which gives the differential structure of the space. It allows to define a generalized, metric compatible, and torsionfree connection $[22,23]$. Moreover, a generalized Ricci tensor, whose flatness condition reproduces the supergravity equations of motion, and a generalized Ricci scalar, encoding the supergravity Lagrangian [22, 23], can be constructed. For the case of Generalized Complex Geometry, these tensors were worked out in [24-26], inspired from older double formalism [29, 30]-[32]. More explicit constructions in DFT are presented in [33, 34]-[40].

In this paper, we extend the more explicit DFT constructions to the exceptional case, for $d=6$, when the symmetry group is ${ }^{1} E_{7}$, following the definitions introduced in [22, 23] for EGG. To be more precise, from the generalized Lie derivative acting on the bein of the generalized metric we define the "dynamical fluxes". These are required to live in certain representations of the duality group. Jacobi-type constraints on these fluxes are obtained from requiring closure of the algebra and gauge invariance. Interestingly enough, for constant fluxes, which correspond to the embedding tensor of $N=8$ supergravity [41], the Jacobi-type constraints reduce to the usual quadratic constraints of maximal supergravity. We show that a generalized Levi-Civita connection, related to the fluxes by a torsion free condition, can be constructed allowing to build Riemann and Ricci tensors [22, 23]. When

\footnotetext{
${ }^{1}$ All throughout the paper when we refer to $E_{7}$, we really mean $E_{7(7)}$.
} 
these are defined in the usual way, they turn out to be non-tensorial, and thus one has to resort to generalized versions of them. Covariant definitions of these tensors were introduced in $[22,23,33,34,36,37]$, which interestingly contain undetermined components. Here, extending the definition of $[33,34]$ to the exceptional case, we find a covariant (though still not uniquely defined) version of the generalized Ricci tensor. Taking its trace, the undetermined pieces go away, and we show that the generalized Ricci scalar, which coincides with that of $[22,23]$ when the section condition is imposed, can be written purely in terms of the dynamical fluxes. In the case of constant fluxes, the generalized Ricci scalar is exactly equal to the potential of $N=8$ supergravity, provided we identify the generalized metric with the moduli space metric for the $N=8$ scalars. Some definitions and results for $d<6$ are given in [27], and some more for $d=4,5$ in [42]. Finally, for completion we provide a list of complementary results along these lines [43-45]-[51].

The paper is organized as follows. In section 2 we set the basic notation and address the definition of generalized diffeomorphisms on a 56 dimensional extended space. The generalized Lie derivative is introduced and through it the "dynamical fluxes" are defined. In section 3 we deal with constraints required from closure of the algebra of gauge transformations. We discuss two different solutions to the Jacobi-type constraints. The so called section condition (also known as strong constraint) and what we call twisted constraints. We mainly deal with these latter constraints. They reproduce the quadratic constraints of maximal $N=8$ supergravity and would naturally appear in Scherk-Schwarz like compactifications. A geometrical structure for the extended space is addressed in section 4 where a generalized covariant derivative and generalized torsion are introduced. A generalized Ricci tensor is defined in section 5. Interestingly enough it is shown that, under contractions with the generalized metric, the associated Ricci scalar is completely determined in terms of generalized fluxes, and corresponds to the scalar potential of maximal supergravity. Final comments are presented in section 6 . Some notation and useful results are summarized in the appendices.

\section{Generalized diffeomorphisms and fluxes}

Our starting point is the $\mathbf{5 6}$-dimensional exceptional generalized tangent space, or extended space, for an extended version of Double Field Theory, where 56 is the dimension of the fundamental representation of $E_{7}$. For toroidal compactifications, such a space was called megatorus [14]. ${ }^{2}$ This space encodes all the symmetries of string theory compactified on 6-manifolds, or M-theory compactified on 7-manifolds, namely internal diffeomorphisms and gauge transformations of the NSNS and RR gauge fields (or the 3-form gauge field in M-theory).

The coordinates in the extended space are $Y^{M}$ with $M=1, \ldots, 56$, and the derivatives are noted by $\partial_{M}=\partial / \partial Y^{M}$ and transform in the fundamental representation of $E_{7}$. We postpone to section 3 the discussion about constraints to be satisfied by them.

\footnotetext{
${ }^{2}$ Here we do not require the extended space to be a parallelizable manifold (i.e., a torus or a "twisted torus"), except for sections 3.2 and 5.2, and at the very end of section 5, where we compare the generalized Ricci scalar to the potential of $N=8$ supergravity.
} 
We will actually consider an augmented duality group, $\mathbb{R}^{+} \times E_{7}$, which accounts for a conformal factor [22-26]. This gives an extra degree of freedom in the $4 \mathrm{D}$ supergravity whose string theory origin can be traced to the measure of the 6-, 7-dimensional manifold, as well as extra gauge parameters, as we will see.

There is a generalized metric on the extended space $H^{M N}$, which transforms covariantly under $\mathbb{R}^{+} \times E_{7}$, and is invariant under $S U(8)$, the maximal compact subgroup of $E_{7}$. It can be written in terms of a generalized bein $E_{\bar{A}}{ }^{M}$ taking values in the quotient $\mathbb{R}^{+} \times E_{7} / S U(8)$

$$
E_{\bar{A}}^{M}=e^{-\Delta} \tilde{E}_{\bar{A}}^{M},
$$

where $\tilde{E}_{\bar{A}}^{M}$ is an $E_{7}$ frame, and the conformal factor $e^{-\Delta}$ corresponds to the component in $\mathbb{R}^{+}$. In what follows, the tilde refers to objects that transform under $E_{7}$ only. The generalized metric then reads

$$
H^{M N}=E_{\bar{A}}^{M} E_{\bar{B}}{ }^{N} H^{\bar{A} \bar{B}},
$$

where $\bar{A}, \bar{B}, \ldots=1, \ldots, 56$ are $S U(8)$ planar indices.

The $E_{7}$ generalized bein $\tilde{E}_{\bar{A}}^{M}$ preserves the $S p(56)$ anti-symmetric matrix $\tilde{\omega}_{M N}$

$$
\tilde{E}_{\bar{A}}^{M} \tilde{\omega}_{M N} \tilde{E}_{\bar{B}}^{N}=\tilde{\omega}_{\bar{A} \bar{B}}, \quad \tilde{\omega}_{M N} \tilde{\omega}^{N P}=-\delta_{M}^{P},
$$

so it is natural to define a weighted symplectic metric

$$
\omega_{M N}=e^{2 \Delta} \tilde{\omega}_{M N}
$$

which raises and lowers indices according to the following convention

$$
A^{M}=-\omega^{M N} A_{N}, \quad A_{M}=\omega_{M N} A^{N} .
$$

With this convention, the following relations hold

$$
E^{\bar{A}}{ }_{M} E_{\bar{A}}{ }^{N}=-\delta_{M}^{N}, \quad E^{\bar{A}}{ }_{P} E_{\bar{B}}{ }^{P}=-\delta_{\bar{B}}^{\bar{A}}, \quad \text { where } E^{\bar{A}}{ }_{M}=-\omega^{\bar{A} \bar{B}} \omega_{M N} E_{\bar{B}}{ }^{N} .
$$

In addition to the symplectic invariant, in $\mathbb{R}^{+} \times E_{7}$ there is also a quartic invariant

$$
E^{\bar{A}}{ }_{M} E_{\bar{B}}{ }^{N} E^{\bar{C}}{ }_{P} E_{\bar{D}}{ }^{Q} K^{M}{ }_{N}^{P}{ }_{Q}=K^{\bar{A}}{ }_{\bar{B}}{ }^{\bar{C}}{ }_{\bar{D}} .
$$

A generalized diffeomorphisms compatible with this symmetry group is generated by an infinitesimal generalized vector (or gauge parameter) $\xi$, and is given by the generalized Lie derivative $\mathcal{L}_{\xi}$ (or equivalently a generalized gauged transformation $\delta_{\xi}$, which coincide when acting on tensors). Acting on a generalized vector field $V$, we expect it to be a linear combination of the gauge parameter and its derivatives. A detailed discussion on how to construct generalized diffeomorphisms can be found in appendix C. Here we simply give its general expression

$$
\delta_{\xi} V^{M}=\mathcal{L}_{\xi} V^{M}=\xi^{P} \partial_{P} V^{M}-A^{M}{ }_{N}{ }^{P}{ }_{Q} \partial_{P} \xi^{Q} V^{N}+\frac{\omega}{2} \partial_{P} \xi^{P} V^{M} .
$$


This was first proposed in $[22,23]$ and we are using the notation of [28] (see also [29-31] in DFT context). The tensor $A$ is fixed by requiring that the gauge transformations preserve the $E_{7}$ structure (see (2.3) and (2.7)) and is given by

$$
A^{M}{ }_{N}{ }^{P} Q=12 P_{(a d j)}{ }^{M}{ }_{N}{ }^{P} Q=12\left(t_{\alpha}\right)_{N}{ }^{M}\left(t^{\alpha}\right)_{Q}{ }^{P},
$$

where $t_{\alpha}$ is a generator of $E_{7}$, with $\alpha=1, \ldots, 133$ an index in the adjoint, and $P_{(a d j)}$ is a projection to the adjoint 133 of $E_{7}$. We give its expression in terms of $E_{7}$ invariants in (A.1). The coefficient $\omega$ corresponds to the $\mathbb{R}^{+}$weight of the object being transformed, which for the bein $E_{\bar{A}}{ }^{M}$ is $\omega=1$, but for $\tilde{E}_{\bar{A}}{ }^{M}$ is $\omega=0 .{ }^{3}$

In the appendices we provide more information about the general structure of generalized diffeomorphisms, showing how the $E_{7}$ case arises as a particular example. We also include many useful identities that we will use repeatedly along the paper. The relative coefficient between $A$ and the projector onto the adjoint depends on the group in question. In appendix $\mathrm{C}$ we explain where this arises from, and why it is 12 for the case of $E_{7(7)}$.

Applying the generalized diffeomorphism (2.8) to the bein, generated by a bein itself, we get

$$
\delta_{E_{\bar{A}}} E_{\bar{B}}=F_{\bar{A} \bar{B}} \bar{C} E_{\bar{C}},
$$

where the "generalized dynamical fluxes" $F_{\bar{A} \bar{B}} \bar{C}$ are defined as

$$
F_{\bar{A} \bar{B}} \bar{C}=\Omega_{\bar{A} \bar{B}} \bar{C}-12 P_{(a d j)}{ }_{\bar{B}}^{\bar{D}}{ }_{\bar{E}} \Omega_{\bar{D} \bar{A}} \bar{E}+\frac{1}{2} \Omega_{\bar{D} \bar{A}} \bar{D}_{\bar{B}}^{\bar{C}},
$$

where

$$
\Omega_{\bar{A} \bar{B}}^{\bar{C}}=E_{\bar{A}}^{M} \partial_{M} E_{\bar{B}}^{N}\left(E^{-1}\right)_{N}^{\bar{C}} .
$$

In the case when there is a global frame (or in other words when the space is parallelizable), this object is called the Weitzenböck connection. Here, with the exception of sections 3.2, 5.2 and 5.3, we do not a priori require the existence of such a global frame, and as such our expressions should be understood as local. This means in particular that the generalized dynamical fluxes need not be constant (hence the name dynamical), and furthermore they need not even be globally defined. Nevertheless, by an abuse of notation we will still call these fluxes, and we will call $\Omega$ in (2.12) the Weitzenböck connection.

Rotating these expressions with the bein we can define the fluxes with curved indices

$$
F_{M N}{ }^{P}=\Omega_{M N}{ }^{P}-12 P_{(a d j)}{ }^{P}{ }_{N}{ }_{S} \Omega_{R M}{ }^{S}+\frac{1}{2} \Omega_{R M}{ }^{R} \delta_{N}^{P},
$$

and the corresponding Weitzenböck connection in curved indices

$$
\Omega_{M N}^{P}=\left(E^{-1}\right)_{N}{ }^{\bar{B}} \partial_{M} E_{\bar{B}}^{P} .
$$

The Weitzenböck connection (2.14) takes values in the algebra of $\mathbb{R}^{+} \times E_{7}$ (see appendix B), i.e. it can be written as linear combination of the generators of $\mathbb{R}^{+} \times E_{7}$

$$
\Omega_{M N}^{P}=-\partial_{M} \Delta \delta_{N}^{P}+\tilde{\Omega}_{M N}{ }^{P}=\Omega_{M}^{0}\left(t_{0}\right)_{N}{ }^{P}+\tilde{\Omega}_{M}{ }^{\alpha}\left(t_{\alpha}\right)_{N}{ }^{P},
$$

\footnotetext{
${ }^{3}$ We use the same symbol $\omega$ for the symplectic invariant. However, the latter has two indices, while the weight $\omega$ is a number, so there should be no confusion.
} 
and is therefore in the $\mathbf{5 6} \times(\mathbf{1}+\mathbf{1 3 3})$ product. Here, $\left(t_{0}\right)_{N}{ }^{P}=-\delta_{N}^{P}$ is the generator of $\mathbb{R}^{+}$. The $56 \times 133$ part

$$
\tilde{\Omega}_{M N}{ }^{P}=\left(\tilde{E}^{-1}\right)_{N}{ }^{\bar{B}} \partial_{M} \tilde{E}_{\bar{B}}^{P},
$$

contains the irreducible representations $56+912+\mathbf{6 4 8 0}$. The projectors onto the first two representations in this product is given by (see appendix $\mathrm{C}$ )

$$
\begin{aligned}
P_{(56) A}{ }^{\alpha},{ }_{\beta} & =\frac{56}{133}\left(t^{\alpha} t_{\beta}\right)_{A}{ }^{B} \\
P_{(912) A}{ }^{\alpha},{ }_{\beta} & =\frac{1}{7} \delta_{\beta}^{\alpha} \delta_{A}^{B}-\frac{12}{7}\left(t_{\beta} t^{\alpha}\right)_{A}{ }^{B}+\frac{4}{7}\left(t^{\alpha} t_{\beta}\right)_{A}{ }^{B} .
\end{aligned}
$$

Equations (2.13) to (2.17) imply that the fluxes are in the $\mathbf{9 1 2}$ and $\mathbf{5 6}$ representations only. More precisely

$$
F_{A B}{ }^{C}=X_{A B}^{C}+D_{A B}^{C},
$$

with

$$
X_{A B}^{C}=\Theta_{A}^{\alpha}\left(t_{\alpha}\right)_{B}^{C} \text { with } \Theta_{A}^{\alpha}=7 P_{(912) A^{\alpha}}{ }^{B}{ }_{\beta} \tilde{\Omega}_{B}^{\beta} \text {, }
$$

and

$$
D_{A B}{ }^{C}=-\vartheta_{A} \delta_{B}^{C}+8 P_{(a d j)}{ }^{C}{ }_{B}{ }^{D}{ }_{A} \vartheta_{D}, \quad \vartheta_{A}=-\frac{1}{2}\left(\tilde{\Omega}_{D A}{ }^{D}-3 \partial_{A} \Delta\right) .
$$

The fluxes $F$ involve therefore a projection onto the $\mathbf{9 1 2}$ given by the gaugings $X$ plus contributions from the gaugings $\vartheta$. In the language of gauged supergravity, they correspond to the gauge group generators, i.e. are contractions of the embedding tensor (which dictates how the gauge group is embedded in the global symmetry group) with the generators of the global symmetry group [41]. For this reason we will sometimes call them "gaugings". The $X$ piece in (2.18) corresponds to the $\mathbf{9 1 2}$ component of the fluxes, and in terms of the Weitzenböck connection and the quartic invariant reads

$$
X_{A B C}=\Omega_{A B C}-\Omega_{(B C) A}+12 K_{B C}{ }^{D E} \Omega_{D E A}+\frac{2}{3} \omega_{A(B} \vartheta_{C)}+8 K_{A B C}{ }^{D} \vartheta_{D} .
$$

Using the identities (A.5) of the quartic invariant, one can show that $X$ enjoys the properties of the 912

$$
P_{(a d j)}{ }^{C}{ }_{B}^{D}{ }_{E} X_{A D}{ }^{E}=X_{A B}{ }^{C}, \quad X_{A[B C]}=X_{A B}{ }^{B}=X_{(A B C)}=X_{B A}{ }^{B}=0,
$$

which are the well known conditions satisfied by gaugings in $N=8$ maximal supergravity [54].

The $D$ piece (2.20) contains two terms, one belonging to the $\mathbf{5 6}$ in $\mathbf{5 6} \times \mathbf{1}$, and another belonging to the $\mathbf{5 6}$ in $\mathbf{5 6} \times \mathbf{1 3 3}$. Notice however, that both terms contain the same degrees of freedom in terms of $\vartheta$ and are therefore not independent.

With these results we are able to express the gauge group generators $\left(F_{A}\right)_{B}{ }^{C}$ as in $[57$, $58]$

$$
F_{A}=\vartheta_{A} t_{0}+\left(\Theta_{A}^{\alpha}+8 \vartheta_{B}\left(t^{\alpha}\right)_{A}^{B}\right) t_{\alpha} .
$$

The so-called intertwining tensor (i.e., the symmetric components of the gauge group generators) takes the form

$$
Z_{A B}^{C}=\left(F_{(A}\right)_{B)}{ }^{C}=-\frac{1}{2}\left(\Theta^{C \alpha}-16 \vartheta_{D}\left(t^{\alpha}\right)^{C D}\right)\left(t_{\alpha}\right)_{A B},
$$

and therefore takes values in the $E_{7}$ algebra, as expected $[57,58]$. 


\section{Consistency constraints}

Closure of the algebra and Leibniz rule of gauge transformations

$$
\left[\mathcal{L}_{\xi_{1}}, \mathcal{L}_{\xi_{2}}\right] \xi_{3}^{M}=\mathcal{L}_{\left[\left[\xi_{1}, \xi_{2}\right]\right]} \xi_{3}^{M}=\mathcal{L}_{\mathcal{L}_{\xi_{1}} \xi_{2}} \xi_{3}^{M}
$$

impose a set of Jacobi-type constraints on the vector fields, which are quadratic in derivatives. We first obtain their general expressions, and then show two different set of solutions, commenting on the relevance of each. Defining

$$
\Delta_{123}{ }^{M}=\left[\mathcal{L}_{\xi_{1}}, \mathcal{L}_{\xi_{2}}\right] \xi_{3}^{M}-\mathcal{L}_{\mathcal{L}_{\xi_{1}} \xi_{2}} \xi_{3}^{M}=0
$$

the closure of the algebra and Leibniz rule can be cast in form

$$
\begin{aligned}
\Delta_{[12] 3}{ }^{M} & =\left[\mathcal{L}_{\xi_{1}}, \mathcal{L}_{\xi_{2}}\right] \xi_{3}^{M}-\mathcal{L}_{\left[\left[\xi_{1}, \xi_{2}\right]\right]} \xi_{3}^{M} \\
\Delta_{(12) 3}{ }^{M} & =-\mathcal{L}_{\left(\left(\xi_{1}, \xi_{2}\right)\right)} \xi_{3}^{M}=\mathcal{L}_{\left[\left[\xi_{1}, \xi_{2}\right]\right]} \xi_{3}^{M}-\mathcal{L}_{\mathcal{L}_{\xi_{1}} \xi_{2}} \xi_{3}^{M}
\end{aligned}
$$

where

$$
\left[\left[\xi_{1}, \xi_{2}\right]\right]=\frac{1}{2}\left(\mathcal{L}_{\xi_{1}} \xi_{2}-\mathcal{L}_{\xi_{2}} \xi_{1}\right)
$$

is the Exceptional Courant Bracket $[17,22,23]$, while the $\left(\left(\xi_{1}, \xi_{2}\right)\right)$ is given by

$$
\left(\left(\xi_{1}, \xi_{2}\right)\right)=\frac{1}{2}\left(\mathcal{L}_{\xi_{1}} \xi_{2}+\mathcal{L}_{\xi_{2}} \xi_{1}\right) .
$$

In the following, it is useful to define the invariant [28]

$$
Y^{Q} M^{R}{ }_{S}=\delta_{S}^{Q} \delta_{M}^{R}+\frac{1}{2} \delta_{M}^{Q} \delta_{S}^{R}-12 P_{(a d j)}{ }^{Q} M_{S}^{R}=\frac{1}{2} \omega^{Q R} \omega_{M S}-12 P_{(a d j) M S}{ }^{Q R}
$$

where in the last equality we have used (A.4). The generalized diffeomorphisms (2.8) can be written in terms of this operator as

$$
\mathcal{L}_{\xi} V^{M}=L_{\xi} V^{M}+Y^{M}{ }_{N}{ }^{P}{ }_{Q} \partial_{P} \xi^{Q} V^{N}
$$

where $L_{\xi} V$ is the ordinary Lie derivative. Therefore, $Y$ can be understood as an object measuring the departure from usual Riemannian geometry.

A first constraint arising form closure of the gauge algebra states that two successive gauge transformations should effectively correspond to a unique gauge transformation. If the first (second) transformation is parameterized by $\xi_{1}\left(\xi_{2}\right)$, the parameter of the composed transformation is given by the Exceptional Courant Bracket. Explicitly, this is

$$
\left[\left[\xi_{1}, \xi_{2}\right]\right]^{M}=\xi_{[1}^{P} \partial_{P} \xi_{2]}^{M}-A^{M}{ }_{N}^{P}{ }_{Q} \partial_{P} \xi_{[1}^{Q} \xi_{2]}^{N}+\frac{1}{2} \partial_{P} \xi_{[1}^{P} \xi_{2]}^{M}
$$

Using equation (A.6), the closure condition can be written in the form

$$
\begin{aligned}
\Delta_{[12] 3}{ }^{M}= & Y^{Q}{ }_{L}{ }^{O}{ }_{I} \partial_{O} \xi_{[2}^{I} \xi_{1]}^{L} \partial_{Q} \xi_{3}^{M} \\
& +A^{M}{ }_{N}{ }_{L}{ }_{L} Y^{Q}{ }_{J}{ }^{O}{ }_{I} \partial_{Q} \xi_{[2}^{I} \partial_{O} \xi_{1]}^{L} \xi_{3}^{N} \\
& +Q^{M}{ }_{N}{ }^{Q O}{ }_{L I} \partial_{Q} \partial_{O} \xi_{[2}^{I} \xi_{1]}^{L} \xi_{3}^{N}=0
\end{aligned}
$$


where we have defined

$$
Q^{M}{ }_{N}{ }^{Q O}{ }_{L I}=Y^{Q}{ }_{J}^{O}{ }_{(L} A^{J}{ }_{I}{ }^{M}{ }_{N}+\frac{1}{2} \omega_{I L} Y^{Q M O}{ }_{N}-\frac{1}{2} Y^{Q}{ }_{L}{ }_{I} \delta_{N}^{M} .
$$

Additionally using

$$
\left(\left(\xi_{1}, \xi_{2}\right)\right)^{M}=Y^{M}{ }_{N}{ }^{P}{ }_{Q} \partial_{P} \xi_{(1}^{Q} \xi_{2)}^{N}
$$

the Leibniz rule is written as

$$
\begin{aligned}
-\Delta_{(12) 3}{ }^{M}= & Y^{Q}{ }_{L}{ }_{I} \partial_{Q} \xi_{(1} \xi_{2)}^{L} \partial_{O} \xi_{3}^{M} \\
& -Q^{M}{ }_{N}{ }^{Q O}{ }_{L I} \partial_{Q}\left(\xi_{(1}^{L} \partial_{O} \xi_{2)}^{I}\right) \xi_{3}^{N} \\
& -\frac{1}{4} \omega_{L I} \omega^{Q O} \partial_{Q} \xi_{1}^{L} \partial_{O} \xi_{2}^{I} \xi_{3}^{M}=0
\end{aligned}
$$

Equations (3.9) and (3.12) imply that any theory invariant under generalized diffeomorphisms will necessarily be a constrained or restricted theory, meaning that the fields and gauge parameters will not be generic, but must necessarily obey these differential conditions. Therefore, these constraints select subset of fields and gauge parameters for which the theory can be consistently defined.

Notice also that, as shown in [28], the Jacobiator can be written as

$$
J\left(\xi_{1}, \xi_{2}, \xi_{3}\right) \equiv\left[\left[\left[\left[\xi_{1}, \xi_{2}\right]\right], \xi_{3}\right]\right]+\text { cyclic }=\frac{1}{3}\left(\left(\left[\left[\xi_{1}, \xi_{2}\right]\right], \xi_{3}\right)\right)+\text { cyclic }
$$

so even if non-vanishing, it generates trivial gauge transformations by virtue of (3.12). We would now like to explore two different set of solutions to the above constraints, and comment on their relevance for different purposes.

\subsection{The section condition}

In DFT, there exists a so-called section condition, also known as the strong constraint, consisting of the following differential operator that must annihilate all fields and gauge parameters and their products [3-6]

$$
S=\eta^{m n} \partial_{m} \partial_{n}
$$

where $m, n=1, \ldots, 2 d$ span the fundamental of $O(d, d)$. In the strong version of the constraint, each partial derivative acts on a given field. In its weak version, what should vanish is the second order operator acting on a single field. The result of the strong constraint is that the fields and gauge parameters no longer depend of the full set of $2 d$ coordinates, but rather on a $d$-dimensional section of the space.

The $E_{7}$ version of the constraint is given by the following operator $[22,23]$

$$
S_{M N}=P_{(a d j) M N}{ }^{Q R} \partial_{Q} \partial_{R},
$$

and again in its weak version this whole operator should vanish when acting on a single field, while in its strong version each derivative hits a different field. 
In $S L(8)$ indices $^{4}$ the $\mathbf{6 3}$ and $\mathbf{7 0}$ pieces of the strong version of the constraint $S_{M}{ }^{N}=0$ read (see equation (A.11))

$$
\begin{aligned}
& 0=\partial^{c b} A \partial_{c a} B-\frac{1}{8} \delta_{a}^{b} \partial^{c d} A \partial_{c d} B+\partial^{c b} B \partial_{c a} A-\frac{1}{8} \delta_{a}^{b} \partial^{c d} B \partial_{c d} A \\
& 0=\partial_{[a b} A \partial_{c d]} B+\frac{1}{4 !} \epsilon_{a b c d e f g h} \partial^{e f} A \partial^{g h} B
\end{aligned}
$$

for any pair of fields $A, B$, where $a, b, \ldots=1, \ldots, 8$ are fundamental $S L(8)$ indices. It is not hard to see that implies that the fields can only depend on 7 out of the 56 coordinates of the extended space [28]. Indeed, calling these directions $\hat{a}=1, \ldots, 7$, we get that, up to $E_{7}$ rotations, $\partial_{\hat{a} 8} \equiv \partial_{\hat{a}}, \partial_{\hat{a} \hat{b}}=0, \partial^{a b}=0$ is the only solution to the constraint. This is precisely how the derivative, which spans a 7-dimensional space for compactifications of M-theory on 7-dimensional manifolds, is embedded in the fundamental representation of $E_{7}$ in Exceptional Generalized Geometry [17].

To make contact with DFT, or equivalently with compactifications of type II theories, it is useful to use the $S L(2) \times O(6,6)$ subgroup of $E_{7}$, under which the fundamental representation breaks according to $M=(\hat{\imath} m, \alpha)$ where $\hat{\imath}=+,-(m=1, \ldots, 12)$ is a fundamental of $S L(2)(O(6,6))$, and $\alpha$ is a spinorial index in the $\mathbf{3 2}$ of $O(6,6)$. The 56 coordinates of the extended space contain therefore two copies of the double torus in DFT $\partial_{ \pm m}$ (which are related by an $S L(2)$ S-duality) plus 32 spinorial directions $\partial_{\alpha}$.

The different components of the projector onto the adjoint representation are given in (A.14). In particular, the first one of them gives the following constraint

$$
S_{\hat{\imath} m \hat{\jmath} n}=-\frac{1}{2} \epsilon_{\hat{\imath} \jmath} \partial_{[+\mid m} A \partial_{\mid-] n} B+\frac{1}{12} \eta_{m n} \partial_{(\hat{\imath} \mid p} A \partial_{\mid \hat{\jmath}) q} B \eta^{p q}-\frac{1}{8} \epsilon_{\hat{\imath} \jmath} \partial_{\alpha} A\left[\gamma_{m n}\right]^{\alpha \beta} \partial_{\beta} B .
$$

The requirements $S_{+m+n}=0, S_{-m-n}=0$ give on one hand that the derivative can only span one of the two copies of the DFT coordinates (call it + , or electric), and on the other hand we get exactly the DFT strong constraint (3.14), which implies that the dependence is only on half of the double coordinates, that we call $\bar{m}=1, \ldots, 6$. Using this in the $S_{+m-n}-S_{-m+n}$ constraint, only the last term in (3.17) survives, and one can see that the spinorial derivative can only span a two-dimensional (12-dimensional) subspace in the case of positive (negative) chirality. The former is relevant for compactifications of type IIA, where D-branes have even dimensionality, while the latter applies to type IIB. The allowed components of the spinorial derivatives are further reduced by the condition $S_{+m \alpha}=0$ (where the relevant projector is given in the last line of (A.14)) to a one-dimensional (zerodimensional) space in the case of positive (negative) chirality, defined by the constraint $\left[\gamma^{\bar{m}}\right]_{\alpha}{ }^{\beta} \partial_{\beta}=0 .{ }^{5}$ In summary, the strong constraint implies that the dependence is on 6 coordinates only ${ }^{6} \partial_{+\bar{m}} \neq 0$, plus an extra spinorial coordinate for the case of positive

\footnotetext{
${ }^{4}$ The decomposition of $E_{7}$ representations into $S L(8)$ ones is the same as for $S U(8)$ representations, and these are given in appendix A

${ }^{5}$ In the one-to-one correspondence between $\mathrm{O}(6,6)$ spinors and forms on the cotangent space $T_{6}^{*} M$, this corresponds to the six-forms.

${ }^{6}$ This is precisely how the derivative is embedded into an $E_{7}$ representation for compactifications of type II theories in EGG [61].
} 
chirality, which arises in compactifications of type IIA. This extra coordinate is nothing but the M-theory circle. If one wants to avoid this dependence, an extra constraint should be supplemented to (3.15).

With this information, we can now show that a possibility to solve the constraints (3.9) and (3.12) is to restrict the vector fields to depend only on a 7-dimensional slice of the full mega-space, i.e. constrain them to satisfy the section condition

$$
P_{(a d j) M N}{ }^{P Q} \partial_{P} V^{R} \partial_{Q} U^{S}=0 \quad P_{(a d j) M N}{ }^{P Q} \partial_{P} \partial_{Q} U^{S}=0 .
$$

Notice that in (3.9) and (3.12) all the derivatives are contracted with the $Y$ tensor defined in (3.6). When acting on two partial derivatives $\partial_{Q} \partial_{R}$, the last term on the last equality in (3.6) vanishes due to the section condition. The first term vanishes because the section condition restricts the derivatives to lie in an isotropic bundle, i.e. to have zero inner product with respect to the symplectic form. Therefore the section condition implies

$$
Y_{M}^{Q}{ }_{S} \partial_{Q} \partial_{R}=0
$$

acting on any two fields, and this in turn guarantees that (3.9) and (3.12) are satisfied.

\subsection{Twisted constraints}

In $[12,13]$ it was shown that the section condition is sufficient for the gauge consistency of the theory, but not necessary. Rather, in particular setups such as the ones discussed below, it is not hard to see that the constraints (3.9) and (3.12) are weaker than the strong constraint, and this allows for extended configurations not solving the section condition.

These weaker conditions apply when the vector fields are required to take the form

$$
V^{M}=E_{\bar{A}}^{M}(Y) v^{\bar{A}}
$$

with $v$ constant. Note that this is only possible in parallelizable manifolds, so in this section (as well as in section 5.2), where we use these weaker constraints, we restrict to this case (i.e. we demand that the extended space is parallelizable). This definition can be trivially extended to tensors with more indices. This kind of behavior arises naturally in the context of Scherk-Schwarz compactifications, where the bein plays the role of a twist, and the $v^{\bar{A}}$ vectors correspond to fields or gauge parameters in the effective action, and therefore only depend on space-time coordinates (which we are ignoring in this paper, thus $v$ is constant here). We therefore call the constraints obtained in thee setups "twisted constraints". We will show that requiring the vector fields in planar indices to be constant, equations (3.9) and (3.12) admit solutions that do not necessarily satisfy the section condition.

Let us now move to closure (3.9) and the Leibniz rule (3.12). Notice that in the particular case when (3.9) is evaluated on frames, i.e. $\xi_{1} \rightarrow E_{\bar{A}}, \xi_{2} \rightarrow E_{\bar{B}}$ and $\xi_{3} \rightarrow E_{\bar{C}}$

$$
\begin{aligned}
\Delta_{[\bar{A} \bar{B}] \bar{C}}{ }^{M}= & Y^{Q}{ }_{L}^{O}{ }_{I} \partial_{O} E_{[\bar{B}}^{I} E_{\bar{A}]}^{L} \partial_{Q} E_{\bar{C}}{ }^{M} \\
& +A^{M}{ }_{N}{ }^{J}{ }_{L} Y^{Q}{ }_{J}{ }_{I} \partial_{Q} E_{[\bar{B}}{ }^{I} \partial_{O} E_{\bar{A}]}{ }^{L} E_{\bar{C}}{ }^{N} \\
& +Q^{M}{ }_{N}{ }^{Q O}{ }_{L I} \partial_{Q} \partial_{O} E_{[\bar{B}}^{I} E_{\bar{A}]}{ }^{L} E_{\bar{C}}{ }^{N}
\end{aligned}
$$


and the same for $(3.12)$

$$
\mathcal{L}_{\left(\left(E_{\bar{A}}, E_{\bar{B}}\right)\right)} E_{\bar{C}}{ }^{M}=-\Delta_{(\bar{A} \bar{B}) \bar{C}}{ }^{M} .
$$

Alternatively one can compute $\Delta_{\bar{A} \bar{B} \bar{C}}{ }^{M}$ using (2.10) directly in terms of $F_{\bar{A} \bar{B}} \bar{C}$

$$
\begin{aligned}
\Delta_{\bar{A} \bar{B} \bar{C}} \bar{D}= & \left(\left[F_{\bar{A}}, F_{\bar{B}}\right]+F_{\bar{A} \bar{B}} \bar{E}^{\bar{E}} F_{\bar{E}}\right)_{\bar{C}} \bar{D}^{\bar{D}} \\
& +2 \partial_{[\bar{A}} F_{\bar{B}] \bar{C}} \bar{D}^{\bar{D}}+A^{\bar{D}} \bar{C}_{\bar{C}} \bar{E}_{\bar{F}} \partial_{\bar{E}} F_{\bar{A} B}{ }^{\bar{F}}-\frac{1}{2} \partial_{\bar{E}} F_{\bar{A} \bar{B}} \bar{E}^{\bar{E}} \delta_{\bar{C}}^{\bar{D}} .
\end{aligned}
$$

Then the closure and the Leibniz rule are recovered in the form

$$
\Delta_{\bar{A} \bar{B} \bar{C}}^{M}=0 .
$$

Notice that if the section condition is imposed the closure and the Leibniz rule are guaranteed as noted above. However if we restrict our vectors to take the form (3.20) a weaker version of the constraints can be considered provided the quadratic constraint (3.23) is imposed to ensure the consistency of the theory. In the particular case in which the fluxes are constant, condition (3.23) becomes

$$
\left[F_{A}, F_{B}\right]=-F_{A B}^{C} F_{C}
$$

which are precisely the quadratic constraints of maximal supergravity, even with local scaling symmetry $[57,58]$ (notice that the trace of this equation implies in turn $F_{A B}{ }^{C} \vartheta_{C}=0$ ).

Let us mention that when the fluxes are expressed in planar indices, they should transform as scalars. In fact, we find

$$
\delta_{\xi} F_{\bar{N} \bar{P}}^{\bar{M}}=\xi^{\bar{Q}} \partial_{\bar{Q}} F_{\bar{N} \bar{P}}{ }^{\bar{M}}-\xi^{\bar{Q}} \Delta_{\bar{Q} \bar{N} \bar{P}}{ }^{\bar{M}}
$$

This actually guarantees that $X$ and $\vartheta$ transform as scalars independently. For example, in the case of $\vartheta$ we get

$$
\delta_{\xi} \vartheta_{\bar{M}}=\xi^{\bar{N}} \partial_{\bar{N}} \vartheta_{\bar{M}}-\frac{1}{56} \xi^{\bar{N}} \Delta_{\bar{N} \bar{M} \bar{P}} \bar{P} .
$$

In summary, the constraints (3.24) are the only necessary and sufficient conditions for consistency of the theory at the classical level when the vectors are restricted as in (3.20). So, while the relaxed constraints are necessary and sufficient for gauge consistency in this case, the section condition is only sufficient. In DFT, explicit examples of truly extended configurations were found in [56], and it would be interesting to find some here as well.

A few words are in order. The section condition is crucial to make contact with 10 or 11dimensional supergravity, and therefore puts the extended theory in a safe and controlled place. When relaxed, the connection between this construction and higher dimensional supergravity is less clear and should be understood better. Configurations that satisfy the relaxed constraints but not the section condition lie beyond supergravity compactifications, and are therefore strictly non-geometric (we refer to [56] for a discussion on these issues). Whether they correspond to allowed configurations in the full string or M-theory is a question that remains partially unanswered and worth exploring. 


\section{A geometry for the extended space}

In this section we discuss the covariant derivative on the extended space, seek for a covariant definition of torsion, and propose a set of conditions that the connection must satisfy. Later in the following section we will define a generalized Ricci tensor, and show that under contractions with the generalized metric, the associated Ricci scalar is completely determined in terms of generalized fluxes, and corresponds to the scalar potential of maximal supergravity.

Having defined the generalized notion of Lie derivative in (2.8), it is natural to look for derivatives that behave covariantly under such transformations. We begin by defining the covariant derivative of a bein $E$ as

$$
\nabla_{M} E_{\bar{A}}{ }^{N}=W_{M \bar{A}}{ }^{\bar{B}} E_{\bar{B}}^{N}=\partial_{M} E_{\bar{A}}^{N}+\Gamma_{M P}{ }^{N} E_{\bar{A}}^{P},
$$

in terms of a Christoffel connection $\Gamma$, or alternatively a spin connection $W$. They are related to the Weitzenböck connection $\Omega_{\bar{A}}$ (2.12) taking values in the algebra of $\mathbb{R}^{+} \times E_{7}$ (see appendix B). The three connections are related through

$$
W_{C A}{ }^{B}=\Omega_{C A}{ }^{B}+\Gamma_{C A}{ }^{B} .
$$

In addition, one can relate the gaugings to the Weitzenböck connection through projections, as in equation (2.11).

These connections must also transform properly so as to compensate the failure of the usual derivative to transform as a tensor. Given that the covariant derivative is requested to transform covariantly, so must the spin connection. Hence, taking into account (4.2), we see that the Christoffel connection must fail to transform as minus the failure of the Weitzenböck connection

$$
\Delta_{\xi} \Omega_{B C}{ }^{D}=-\Delta_{\xi} \Gamma_{B C}{ }^{D},
$$

where $\Delta_{\xi}=\delta_{\xi}-\mathcal{L}_{\xi}$ is defined as in an analogous way as in (3.26), and represents the failure of an object to transform as a tensor.

\subsection{Generalized connections and torsion}

We can define the generalized torsion through [24-26]

$$
\mathcal{T}_{\bar{A} \bar{B}}^{\bar{C}} \equiv\left(E^{-1}\right)_{M}^{\bar{C}}\left(\mathcal{L}_{E_{\bar{A}}}^{\nabla}-\mathcal{L}_{E_{\bar{A}}}\right) E_{\bar{B}}{ }^{M},
$$

where $\mathcal{L}^{\nabla}$ is defined as in (2.8), but with a partial replaced by a covariant derivative. Using (4.2) we arrive at

$$
\mathcal{T}_{A B}{ }^{C}=\Gamma_{A B}{ }^{C}-12 P_{(a d j)}{ }^{C}{ }_{B}{ }^{P}{ }_{Q} \Gamma_{P A}{ }^{Q}+\frac{1}{2} \Gamma_{D A}{ }^{D} \delta_{B}^{C} .
$$

A torsionless connection requires this to vanish, which amounts to

$$
\Gamma_{A B}{ }^{C}=12 P_{(a d j)}{ }_{B}{ }^{D}{ }_{E} \Gamma_{D A}{ }^{E}-\frac{1}{2} \delta_{B}^{C} \Gamma_{D A}{ }^{D} .
$$


Now, acting on this condition with the projector to the adjoint in the last two indices, we find

$$
P_{(a d j)}{ }^{G}{ }_{H}^{B}{ }_{C} \Gamma_{A B}{ }^{C}=12 P_{(a d j)}{ }^{G}{ }_{H}^{B}{ }_{C} \Gamma_{B A}{ }^{C},
$$

and now plugging this result in (4.6) we find

$$
\Gamma_{A B}{ }^{C}=P_{(a d j)}{ }_{B}{ }^{D}{ }_{E} \Gamma_{A E}{ }^{D}-\frac{1}{2} \Gamma_{D A}{ }^{D} \delta_{B}^{C},
$$

so the trace of the connection measures its failure to take values in the $\mathbf{5 6} \times \mathbf{1 3 3}$ of $E_{7}$. Notice the two traces of the connection are related

$$
\Gamma_{A B}{ }^{B}=-28 \Gamma_{B A}{ }^{B} .
$$

Let us now dedicate a few lines to comment on the relation between the trace of the Christoffel connection and the $\mathbb{R}^{+} \times E_{7}$ measure. Notice that $\sqrt{H}$ does not transform as a density under the generalized diffeomorphisms (2.8), instead the proper measure is given by $(\sqrt{H})^{-1 / 28}=e^{-2 \Delta}$ since

$$
\delta_{\xi} e^{-2 \Delta}=\partial_{P}\left(e^{-2 \Delta} \xi^{P}\right) .
$$

Partial integration of the covariant derivative in the presence of the $\mathbb{R}^{+} \times E_{7}$ density $e^{-2 \Delta}$

$$
\int e^{-2 \Delta} U \nabla_{M} V^{M}=-\int e^{-2 \Delta} V^{M} \nabla_{M} U
$$

is ensured if

$$
\Gamma_{M N}{ }^{M}=-2 \partial_{N} \Delta .
$$

which, as we show below, is a consequence of metric compatibility. The torsionless (4.6) and the trace equations (4.12) imply that the connection takes the form

$$
\Gamma_{M N}{ }^{P}=\tilde{\Gamma}_{M N}{ }^{P}-\frac{24}{19} P_{(a d j)}{ }^{P}{ }_{N}{ }^{K}{ }_{M} \partial_{K} \Delta+\partial_{M} \Delta \delta_{N}^{P},
$$

where $\tilde{\Gamma}$ belongs to the $\mathbf{6 4 8 0}$ of $\mathbf{5 6} \times \mathbf{1 3 3}$ (which is traceless). This piece remains undetermined here, but a part of it will be fixed by imposing metric compatibility.

We now turn to the analysis of the spin connection $W$. Using (4.2), (2.15) and (4.13) one can obtain

$$
W_{A B}{ }^{C}=\tilde{\Omega}_{A B}{ }^{C}+\tilde{\Gamma}_{A B}{ }^{C}-\frac{24}{19} P_{(a d j)}{ }^{C}{ }_{B}{ }^{P}{ }_{A} \partial_{P} \Delta=\left(\tilde{\Omega}_{A}{ }^{\alpha}+\tilde{\Gamma}_{A}{ }^{\alpha}-\frac{24}{19}\left(t^{\alpha}\right)_{A}{ }^{P} \partial_{P} \Delta\right)\left(t_{\alpha}\right)_{B}{ }^{C} .
$$

From this expression we learn on the one hand that the spin connection takes values in $\mathbf{5 6} \times \mathbf{1 3 3}$, and on the other that its trace is proportional to the $\vartheta$ gaugings $(2.13)$

$$
W_{A B}{ }^{A}=-2 \vartheta_{B} .
$$

Knowing that the spin connection belongs to the $56 \times \mathbf{1 3 3}$, we can now act with the projectors to its irreducible representations, and find

$$
\begin{aligned}
P_{(912) A B}{ }^{C},{ }^{M N}{ }_{P} W_{M N}{ }^{P} & =\frac{1}{7} X_{A B}{ }^{C} \\
P_{(56) A B}{ }^{C},{ }^{M N}{ }_{P} W_{M N}{ }^{P} & =-\frac{16}{19} P_{(a d j)}{ }^{C}{ }_{B}{ }^{D}{ }_{A} \vartheta_{D},
\end{aligned}
$$


while the projector to the $\mathbf{6 4 8 0}$ just relates it to $\tilde{\Gamma}$ which is undetermined by the torsionless condition. Finally let us write explicitly the expression for the torsionless condition in terms of $W$, because it will be useful in the following

$$
W_{A B}^{C}-12 P_{(a d j)}{ }^{C}{ }_{B}^{D}{ }_{E} W_{D A}^{E}=F_{A B}^{C}+\vartheta_{A} \delta_{B}^{C} .
$$

\subsection{Generalized metric compatibility}

Another condition we can impose to the connections is compatibility with the generalized metric $H$, equation (2.2). The constraint reads

$$
0=\nabla_{A} H^{B C} \quad \Rightarrow \quad W_{A}^{\alpha}\left(t_{\alpha}\right)_{\bar{D}}^{(\bar{B}} H^{\bar{C}) \bar{D}}=0 .
$$

It is instructive to turn to the $S U(8)$ language. When $E_{7}$ is broken to $S U(8)$, the fundamental $\mathbf{5 6}$ and adjoint $\mathbf{1 3 3}$ representations break according to

$$
M=\mathbf{5 6}=\mathbf{2 8}+\overline{\mathbf{2 8}}=\left(M_{[i j]}, M^{[i j]}\right), \quad \alpha=\mathbf{1 3 3}=\mathbf{6 3}+\mathbf{7 0}=\left(\alpha_{i}^{j}, \alpha_{[i j k l]}\right),
$$

where $i, j, \cdots=1, \ldots, 8$ and the $\mathbf{6 3}$ is traceless $\alpha_{i}{ }^{i}=0$. One can then see that metric compatibility equations (4.18) reduce to

$$
W_{A}^{[i j k l]}=0
$$

Hence the projection of the spin connection to the $\mathbf{7 0}$ of $\mathbf{1 3 3}$ must vanish. On the other hand, metric compatibility leaves the $\mathbf{6 3}$ piece of the connection undetermined.

Another consequence of the metric compatibility is eq. (4.12). This can be proven taking into account (4.9), the fact that the metric is covanriantly constant (eq. (4.18)) and the useful formula for the derivative of the determinat

$$
\partial_{A} H=-H H_{B C} \partial_{A} H^{B C}, \quad H \equiv \operatorname{det} H_{A B}=e^{2 \cdot 56 \Delta}
$$

Let us also comment on compatibility with $E_{7}$ invariants. The compatibility with the symplectic metric is not an additional constraint on the connection, but is automatically satisfied, in fact

$$
\nabla_{M} \omega^{P Q}=-2 \partial_{M} \Delta \omega^{P Q}-2 \Gamma_{M}^{[P Q]}=0
$$

where we have used (4.13). The same holds for the compatibility with the quartic invariant, since

$$
\nabla_{M} K^{P Q R S}=-4 \partial_{M} \Delta K^{P Q R S}+4 \Gamma_{M L}^{(P} K^{Q R S) L}=0
$$

is automatically satisfied using (A.7).

We summarize the properties of the different connections introduced, as well as that of the fluxes, in table 1. 


\begin{tabular}{|l|c|c|c|c|}
\hline & $\Omega$ & $F$ & $W$ & $\Gamma$ \\
\hline Name & Weitzenböck & Fluxes & Spin & Levi-Civita \\
\hline Definition & $\left(E^{-1}\right)_{N} \bar{A} \partial_{M} E_{\bar{A}}{ }^{P}$ & $\Omega_{M N}{ }^{P}-A^{P}{ }_{N}{ }^{R} \Omega_{R M}{ }^{S}+\frac{1}{2} \Omega_{Q M}{ }^{Q} \delta_{N}^{P}$ & $\Omega+\Gamma$ & $\nabla-\partial$ \\
\hline $\mathbb{R}^{+} \times E_{7}$ Rep & $\mathbf{5 6}+\mathbf{9 1 2}+\mathbf{6 4 8 0}$ & $\mathbf{5 6}(D)+\mathbf{9 1 2}(X)$ & $\begin{array}{c}(\mathbf{5 6}+\mathbf{9 1 2})(F) \\
+\mathbf{6 4 8 0}\end{array}$ & $\mathbf{5 6}+\mathbf{6 4 8 0}(\tilde{\Gamma})$ \\
\hline $\begin{array}{l}\text { Undetermined } \\
\text { (torsionless) }\end{array}$ & ---- & ---- & $\mathbf{6 4 8 0}$ \\
\hline $\begin{array}{l}\text { Undetermined } \\
\text { (Metric comp.) }\end{array}$ & ---- & ---- & $\mathbf{5 6} \times \mathbf{6 3}$ \\
\hline
\end{tabular}

Table 1. Definitions and properties of the different connections introduced. In parenthesis we have indicated the name given to the particular representations. "Undetermined (torsionless/Meric comp.)" means that the given component is not fixed by the torsionless/metric compatibility condition.

\section{Generalized Ricci tensor and the scalar potential of gauged maximal supergravity}

The Riemann and torsion tensors are usually defined through the relation

$$
\left[\nabla_{M}, \nabla_{N}\right] V_{P}=-R_{M N P}{ }^{L} V_{L}-T_{M N}{ }^{L} \nabla_{L} V_{P}
$$

with

$$
R_{M N P}^{R}=\partial_{M} \Gamma_{N P}^{R}-\partial_{N} \Gamma_{M P}^{R}+\Gamma_{M L}^{R} \Gamma_{N P}^{L}-\Gamma_{N L}^{R} \Gamma_{M P}^{L},
$$

and

$$
T_{M N}^{P}=\Gamma_{M N}^{P}-\Gamma_{N M}^{P} .
$$

We already discussed a generalized version of torsion, arguing that the usual definition is non-covariant under generalized diffeomorphisms (2.8). The same happens to the Riemann tensor and its trace (the Ricci tensor), and then one has to resort to generalized versions of them. We will now split the discussion in two parts. We will begin with the definition of a generalized Ricci tensor, that is covariant under generalized diffeomorphisms that close under the section condition. Then, we will extend the definition of this tensor so that it is also covariant under generalized diffeomorphisms that close under the twisted constraints.

\subsection{Generalized Ricci tensor and the section condition}

In this section we will restrict to diffeomorphisms parameterized by vectors obeying the section condition, defined in section 3.1 .

As we mentioned, under generalized diffeomorphisms (2.8), the generalized Riemann tensor is not covariant. Using the section condition (3.15) (or its variant (3.19)), we find

$$
\Delta_{\xi} R_{M N K}{ }^{L}=2 \Delta_{\xi} \Gamma_{[M N]}^{Q} \Gamma_{Q K}^{L}, \quad \Delta_{\xi} \Gamma_{M P}{ }^{N}=12 P_{(a d j)}{ }^{N}{ }_{P}{ }^{R}{ }_{S} \partial_{M} \partial_{R} \xi^{S}-\frac{1}{2} \partial_{M} \partial_{Q} \xi^{Q} \delta_{N}^{P},
$$

which in the usual case (i.e. in ordinary general relativity where $Y=0$ ) vanishes due to vanishing torsion. Notice that for everything to be consistent here, we must have 
$\Delta_{\xi} \Gamma_{N M^{N}}=-2 \Delta_{\xi}\left(\partial_{M} \Delta\right)$, and this holds up to terms that vanish under the section condition.

The usual Ricci tensor is defined as

$$
R_{M N}=R_{M P N}^{P},
$$

and in this case is not symmetric, and fails to transform covariantly as

$$
\Delta_{\xi} R_{M N}=2 \Delta_{\xi} \Gamma_{[M Q]}^{P} \Gamma_{P N}^{Q} .
$$

However, note that the vanishing (generalized) torsion condition (4.6) imposes

$$
2 \Gamma_{[M N]}^{Q}=-Y^{Q}{ }_{N}^{R}{ }_{P} \Gamma_{R M}^{P},
$$

and this allows to rewrite

$$
2 \Delta_{\xi} \Gamma_{[M P]}^{Q} \Gamma_{Q N}^{P}=2 \Delta_{\xi} \Gamma_{P M}^{Q} \Gamma_{[N Q]}^{P} .
$$

Using this, is it easy to see that the following symmetric object

$$
\mathcal{R}_{M N} \equiv \frac{1}{2}\left(R_{M N}+R_{N M}+\Gamma_{R M}{ }^{P} Y_{P}^{R}{ }_{Q}{ }_{Q} \Gamma_{S N}{ }^{Q}\right)=\mathcal{R}_{N M},
$$

is a covariant extension of the Ricci tensor

$$
\Delta_{\xi} \mathcal{R}_{M P}=0
$$

This is the natural extension of the DFT definition of Ricci tensor introduced in $[33,34$, $36,37]$.

Let us conclude this section by noticing that a definition of the generalized Ricci tensor can be given in terms of covariant derivatives. In fact, after some algebra we find that $[22,23]$

$$
\left[\nabla_{M}, \nabla_{P}\right] V^{P}+\frac{1}{2} \nabla_{A}\left(Y^{A}{ }_{M}{ }^{B}{ }_{P} \nabla_{B} V^{P}\right)=\mathcal{R}_{M R} V^{R} .
$$

Namely, the generalized Ricci tensor can be expressed as a commutator of covariant derivatives plus a term proportional to the invariant $Y$ that, as pointed out above, measures in some sense the departure from ordinary Riemannian geometry. Interestingly enough, due to the section condition the operator $\nabla_{A}\left(Y^{A}{ }_{M}{ }^{B}{ }_{P} \nabla_{B} \cdot\right)$ has no second order derivatives. Moreover, when the Ricci is projected to the space of deformations of the generalized metric, its undetermined pieces get projected out [22, 23].

\subsection{Generalized Ricci tensor and twisted conditions}

In this section we assume that all vectors take the form (3.20), and consider diffeomorphisms that close under the twisted constraints of section 3.2.

The starting point is the failure of the Christoffel connection to transform as a tensor

$$
\Delta_{\xi} \Gamma_{M P}{ }^{Q}=A_{P}^{Q}{ }_{P}^{R} \partial_{M} \partial_{R} \xi^{S}-\frac{1}{2} \delta_{P}^{Q} \partial_{M} \partial_{R} \xi^{R}-Y^{N}{ }_{M}^{R}{ }_{S} \Omega_{R L}{ }^{S} \Omega_{N P}{ }^{Q} \xi^{L} .
$$


It can be verified that its trace (4.12) transforms properly, provided the twisted constraints hold

$$
\Delta_{\xi} \Gamma_{N M}{ }^{N}-\Delta_{\xi}\left(-2 \partial_{M} \Delta\right)=-\frac{1}{28} \xi^{P} \Delta_{P M N}{ }^{N}=0 .
$$

In this case, for the Riemann tensor we get

$$
\begin{aligned}
\Delta_{\xi} R_{M N K}{ }^{L}= & \left(2 \Delta_{E_{\bar{A}}} \Gamma_{[M N]}{ }^{Q} \Gamma_{Q K}{ }^{L}\right. \\
& -2 Y^{T}{ }_{[M \mid}{ }_{S} \partial_{R} E_{\bar{A}}{ }^{S} \partial_{T} \Gamma_{\mid N] K}{ }^{L}-2 Y^{T}{ }_{[M \mid}{ }^{R}{ }_{S} \partial_{\mid N]}\left(\Omega_{R \bar{A}}{ }^{S} \Omega_{T K}{ }^{L}\right) \\
& \left.-2 Y^{T}{ }_{\left[\left.M\right|^{R}\right.}^{R} \Omega_{R \bar{A}}{ }^{S}\left(\Omega_{T K}{ }^{O} \Gamma_{\mid N] O}{ }^{L}-\Omega_{T \mid N]}{ }^{O} \Gamma_{O K}{ }^{L}-\Omega_{T O}{ }^{L} \Gamma_{\mid N] K}{ }^{O}\right)\right) \xi^{\bar{A}} .
\end{aligned}
$$

So again we find that the usual Riemann tensor (i.e. in ordinary general relativity) is not covariant under the diffeomorphisms that close under the twisted constraints. The same happens for the usual Ricci tensor, because tracing the above expression does not solve the problem

$$
R_{M N}=R_{M P N}^{P}, \quad \Delta R_{M N}=\Delta R_{M P N}^{P} \neq 0 .
$$

However, the Ricci tensor can be generalized into a (symmetric) generalized Ricci tensor by slightly extending the definition (5.9)

$$
\mathcal{R}_{M N} \equiv \frac{1}{2}\left(R_{M N}+R_{N M}+\Gamma_{R M}{ }^{P} Y^{R}{ }_{P}{ }^{S}{ }_{Q} \Gamma_{S N}{ }^{Q}-\Omega_{R M}{ }^{P} Y^{R}{ }_{P}{ }^{S}{ }_{Q} \Omega_{S N}{ }^{Q}\right)=\mathcal{R}_{N M} .
$$

The last term vanishes if the section condition is imposed, which is not the case here. This term must therefore be added to define a covariant Ricci tensor. The trace of this term is the analogue of the term added in DFT in $[12,13]$.

To check the covariance of the generalized Ricci tensor up to twisted constraints, it is instructive to use planar indices. We first write the Riemann tensor in planar indices

$$
R_{\bar{C} \bar{D} \bar{A}}{ }^{\bar{B}}=2 \partial_{[\bar{C}} W_{\bar{D}] \bar{A}} \bar{B}^{\bar{B}}-2 \Omega_{[\bar{C} \bar{D}]}{ }^{\bar{E}} W_{\bar{E} \bar{A}}{ }^{\bar{B}}-2 W_{[\bar{C} \mid \bar{A}}{ }^{\bar{E}} W_{\mid \bar{D}] \bar{E}}{ }^{\bar{B}},
$$

in terms of which the generalized Ricci tensor (5.16) takes the form

$$
2 \mathcal{R}_{\bar{A} \bar{B}}=R_{\bar{A} \bar{D} \bar{B}}^{\bar{D}}+R_{\bar{B} \bar{D} \bar{A}} \bar{D}^{\bar{D}}+(W-\Omega)_{\bar{D} \bar{A}}{ }^{\bar{E}} Y^{\bar{D}} \overline{\bar{E}}_{\bar{G}}^{\bar{F}}(W-\Omega)_{\bar{F} \bar{B}}{ }^{\bar{G}}-\Omega_{\bar{D} \bar{A}} \bar{E}^{\bar{D}} Y_{\bar{E}}^{\bar{D}} \bar{G}_{\bar{G}} \Omega_{\bar{F} \bar{B}}{ }^{\bar{G}} .
$$

Using the following identity

$$
Y^{\bar{D}} \bar{E}_{\bar{E}}^{\bar{F}} \Omega_{\bar{F} \bar{B}}^{\bar{G}}=F_{\bar{B} \bar{E}} \bar{D}-2 \Omega_{[\bar{B} \bar{E}]} \bar{D},
$$

the generalized Ricci tensor can be recast in the form

$$
\begin{aligned}
2 \mathcal{R}_{\bar{A} \bar{B}}= & 2 W_{\bar{E}(\bar{A}}{ }^{\bar{D}}(W-F)_{\bar{B}) \bar{D}}{ }^{\bar{E}}+W_{\bar{D} \bar{A}} \bar{E}^{\bar{D}}{ }_{\bar{E}} \bar{F}_{\bar{G}} W_{\bar{F} \bar{B}}{ }^{\bar{G}}-2 W_{\bar{D} \bar{E}}{ }^{\bar{D}} W_{(\bar{A} \bar{B})} \bar{E} \\
& -2 \partial_{\bar{D}} W_{(\bar{A} \bar{B})} \bar{D}+2 \partial_{(\bar{A} \mid} W_{\bar{D} \mid \bar{B})}^{\bar{D}} .
\end{aligned}
$$

Here, the first line is manifestly covariant, because (the planar version of) both the spin connection and the fluxes are covariant, up to the twisted constraints. The covariance in the second line is less trivial, because derivatives of scalars are only tensors when the section condition holds

$$
\Delta_{\xi}\left(\partial_{M} \phi\right)=Y^{R}{ }_{M}^{P}{ }_{Q} \partial_{P} \xi^{Q} \partial_{R} \phi
$$


which is not an assumption in this section. Notice however, that the last two terms can be re-written in an explicitly covariant form

$$
-2 \partial_{\bar{D}} W_{(\bar{A} \bar{B})}^{\bar{D}}+2 \partial_{(\bar{A} \mid} W_{\bar{D} \mid \bar{B})}^{\bar{D}}=2 W_{\bar{Q} \bar{N}}{ }^{\bar{Q}} W_{(\bar{A} \bar{B})}{ }^{\bar{N}}-8 \vartheta_{\bar{A}} \vartheta_{\bar{B}}-2 \nabla_{N} V_{(\bar{A} \bar{B})}^{N},
$$

where $V^{N}(\bar{A} \bar{B})$ is a tensor defined by

$$
V_{(\bar{A} \bar{B})}^{N}=E_{\bar{C}}^{N} W_{(\bar{A} \bar{B})}^{\bar{C}}+2 E_{(\bar{A}}^{N} \vartheta_{\bar{B})} .
$$

We finish this section by giving an explicit expression for a covariant generalized Ricci tensor

$$
2 \mathcal{R}_{\bar{A} \bar{B}}=2 W_{\bar{E}(\bar{A}} \bar{D}(W-F)_{\bar{B}) \bar{D}}{ }^{\bar{E}}+W_{\bar{D} \bar{A}}{ }^{\bar{E}} Y^{\bar{D}}{ }_{\bar{E}} \bar{F}{ }_{\bar{G}} W_{\bar{F} \bar{B}} \bar{G}-8 \vartheta_{\bar{A}} \vartheta_{\bar{B}}-2 \nabla_{N} V^{N}{ }_{(\bar{A} \bar{B})}
$$

When tracing this expression and integrating with the measure to obtain an action, the last term gives a total derivative and therefore vanishes. We emphasize that this generalized Ricci tensor was constructed only imposing the twisted constraints, and reduces to (5.9) if the section condition is imposed.

\subsection{Generalized Ricci scalar and scalar potential of maximal supergravity}

As we show in the appendix D, when taking the trace of the generalized Ricci tensor (5.16) with the generalized metric $H$

$$
\mathcal{R}=H^{\bar{A} \bar{B}} \mathcal{R}_{\bar{A} \bar{B}},
$$

the undetermined pieces of the connections drop out, and it can be expressed purely in terms of fluxes (no constraints are imposed in this derivation). In the particular case of $\vartheta_{A}=0$, we find

$$
\frac{1}{4} \mathcal{R}=\frac{1}{672}\left(H^{A D} H^{B E} H_{C F} X_{A B}^{C} X_{D E}^{F}+7 H^{A B} X_{A C}{ }^{D} X_{B D}{ }^{C}\right) .
$$

Remarkably, this takes the exact same form as the scalar potential of gauged maximal supergravity [54] if we identify the generalized metric with the moduli space matrix $\mathcal{M}$. Note also that this is true for any torsionless and metric-compatible connection, and the concrete expression of the determined part does not need to be known. In fact, we never needed to solve for the spin connection, but only used the equation that defines it implicitly.

Finally, notice that by definition the Ricci scalar transforms indeed as a scalar under generalized diffeomorphisms

$$
\delta_{\xi} \mathcal{R}=\xi^{P} \partial_{P} \mathcal{R}
$$

This can also be checked taking into account that the fluxes are covariant provided the quadratic constraints hold. Combining this with the fact that $e^{-2 \Delta}$ transforms as a density (4.10)

$$
\delta_{\xi} e^{-2 \Delta}=\partial_{P}\left(e^{-2 \Delta} \xi^{P}\right),
$$

we arrive at the action of EFT

$$
S=\frac{1}{4} \int d^{56} Y e^{-2 \Delta} \mathcal{R}
$$


which is invariant under generalized diffeomorphisms (2.8). In appendix D we provide a detailed derivation of (5.26). Form (5.29) we can see that in the context of string theory or $\mathrm{M}$ theory, when the section condition holds, $e^{-2 \Delta}$ can be identified with the measure of the internal 6 or 7-dimensional manifold

$$
e^{-2 \Delta} \propto \sqrt{g}
$$

\section{Summary and outlook}

In this work we explored the U-duality covariant framework of extended geometry, focusing on the case of $E_{7}$, and applied it to describe the moduli space of maximal gauged supergravity in four dimensions. The extended space is a $\mathbf{5 6}$-dimensional mega-space equipped with a generalized bein taking values in $\mathbb{R}^{+} \times E_{7} / S U(8)$, which can be parameterized in terms of Type II or M-theory degrees of freedom. The first step in the construction is the introduction of generalized gauge transformations (or generalized diffeomorphisms) (2.8), which unify all the possible gauge transformations of the theory inherited from the metric, NSNS and RR forms in Type II strings or from the 3-form in M-theory.

When the generalized diffeomorphisms act on the bein, one obtains field-dependent (i.e. non constant) fluxes (2.11), which are in the $\mathbf{5 6}+\mathbf{9 1 2}$ irreducible representations of $E_{7}$. For consistency, the generalized diffeomorphisms must satisfy a set of conditions, such as closure of the gauge algebra (3.9). We showed that, as happens in DFT, these constrains allow for at least two different type of "solutions". One of them are configurations obeying the section condition (3.18), which implies that the fields only depend on coordinates spanning a 7-dimensional slice of the extended space, therefore allowing to make contact with supergravity and Exceptional Generalized Geometry. In the other type of solutions, which we call "twisted", the fields are taken to have a Scherk-Schwarz form, and the constraints translate into constraints for the fluxes. Interestingly, in the case of constant fluxes they match the quadratic constraints of maximal supergravity, but more generally we provide the extension to the case of non-constant fluxes. The advantage of this second approach is that it allows for truly extended configurations, with dependence on the extra coordinates. Duality orbits of gaugings allowed in maximal gauged supergravity which are beyond those coming from conventional compactifications (and their dual configurations) can be reached in this way.

We then described the geometry of the extended space, starting from a derivative transforming covariantly under generalized diffeomorphisms, and their corresponding Christoffel and spin connections. These are not uniquely defined, only a subset of its components are, via the torsionless and metric compatibility conditions. We summarized the properties of the different connections in table 1. The next question is whether a curvature for the extended space can be defined. Since there seems to be no easy way to define a covariant generalized Riemann tensor [24-26], we considered only the Ricci tensor and scalar, which transform appropriately up to the constraints of the theory. We showed how to meet these conditions in two different ways. In the first one, the Ricci tensor behaves appropriately if the section condition is imposed, and it corresponds to the natural generalization of that 
introduced in the context of Double Field Theory [33, 34, 36, 37] and equals that of $[22,23]$ in the context of generalized $\left(\mathbb{R}^{+} \times E_{7(7)}\right)$ geometry. In the second approach, the section condition is relaxed within the context of Scherk-Schwarz-like compactifications, and we showed that the definition of the Ricci tensor must be further extended so that it is also covariant up to the twisted constraints (3.24). Up to our knowledge, this is the first covariant construction of an extended geometry where the section condition is not imposed.

Finally, we showed that the resulting Ricci scalar matches exactly the scalar potential of gauged maximal supergravity, provided one associates the generalized metric with the moduli scalar matrix, the dynamical fluxes are taken to be the constant gaugings, and the gaugings in the $\mathbf{5 6}$ are taken to zero. Although the original expression for the generalized Ricci scalar (5.16) is a function of the spin connection, which contains undetermined pieces, we show that these contributions simply drop out, and therefore any torsionless and metric compatible connection gives the desired Ricci scalar, whatever its undetermined part is.

Let us finally comment on some interesting questions that remain open, and are worth exploring in our point of view. It is known that the section condition implies that this framework is a covariant re-writing of higher-dimensional supergravity compactifications. In this kind of compactifications only a subset of the gaugings can be reached in the lower dimensional effective action, and thus the space of gauging orbits is split into those that can be obtained (geometric) and those that cannot (non-geometric) [56]. Restricting to Scherk-Schwarz-type backgrounds, this construction does not necessarily use the section condition, and instead uses the twisted conditions, which are in one to one correspondence with the constraints of gauged supergravity. Therefore, any orbit of gaugings can be reached geometrically in this construction, even those that are non-geometric from a supergravity point of view. Recently there has been much progress in moduli-fixing, fluxed induced supersymmetry breaking, de Sitter vacua surveys, etc. in the presence of non-geometric gaugings, and we believe that this framework can shed light on the higher dimensional uplift of these orbits, as background fluxes on the mega-space. For example, recently a one-parameter family of new maximal gauged supergravity with $S O(8)$ gauge group was found [62]. It would be nice to seek for an uplift of these gaugings to the mega-space considered here.

Note added. After our work appeared on the ArXiv the preprint [63], with substantial overlap with our sections 4 and 5, was uploaded.

\section{Acknowledgments}

We are very grateful to C. Nuñez for collaboration in the early stages of this project. We also thank E. Andrés, G. Dibitetto, V. Penas, M. Trigiante and D. Waldram for useful discussions and comments. G.A. thanks the ICTP for hospitality during the completion of this work. This work was partially supported by CONICET, the ERC Starting Grant 259133 - ObservableString and EPLANET. 


\section{A Useful $E_{7}$ identities}

The $e_{7(7)}$ algebra with generators $\left(t_{\alpha}\right)_{M}{ }^{N}$ where $\alpha=1, \ldots, 133$ is an index in the adjoint 133, and $M, N, \cdots=1, \ldots, 56$. The indices are raised and lowered with the symplectic $S p(56) \supset E_{7}$ metric $\omega_{M N}$ according to the conventions (2.5). With this in mind, the adjoint of $E_{7}$ is symmetric $\left(t_{\alpha}\right)_{(M N)}$.

The symplectic metric $\omega_{M N}$ is left invariant by $E_{7}$ transformations, as is the quartic invariant $K_{M N P Q}$. Contracting two generators, we can define a projector to the adjoint representation

$$
P_{(a d j) M N P Q}=\left(t_{\alpha}\right)_{M N}\left(t^{\alpha}\right)_{P Q}=\frac{1}{12} \omega_{M(P} \omega_{Q) N}+K_{M N P Q},
$$

satisfying the useful identities

$$
\begin{aligned}
P_{(a d j)}{ }^{M}{ }_{N}{ }^{P}{ }_{Q} P_{(a d j)}{ }^{Q}{ }_{P}{ }_{S} & =P_{(a d j)}{ }^{M}{ }_{N}{ }_{S}, \quad P_{(a d j)}{ }^{M}{ }_{N}{ }^{N}{ }_{M}=133 \\
P_{(a d j) M N}{ }^{P Q} & =P_{(a d j)(M N)}{ }^{(P Q)}=P_{(a d j)}{ }^{P Q}{ }_{M N},
\end{aligned}
$$

and

$$
P_{(a d j) M}{ }^{K}{ }_{N}^{L}=\frac{1}{24} \delta_{M}^{K} \delta_{N}^{L}+\frac{1}{12} \delta_{M}^{L} \delta_{N}^{K}-\frac{1}{24} \omega_{M N} \omega^{K L}+P_{(a d j) M N}{ }^{K L} .
$$

Also, using (A.2) one can show

$$
K_{M N}{ }^{P Q} K^{M N K L}=-\frac{5}{6} K^{P Q K L}-\frac{11}{12 \times 12} \omega^{P(K} \omega^{L) Q} .
$$

A very useful identity to show the relation between the relaxed constraints and the section condition is

$$
12 P_{(a d j)}{ }^{(M N}{ }_{Q T} P_{(a d j)}{ }^{P) T}{ }_{R S}-4 K^{M N P T} P_{(a d j) T Q R S}+P_{(a d j)}{ }_{R S}^{(M N} \delta_{Q}^{P)}=0,
$$

and the final useful properties we used in the paper are

$$
\left(t_{\beta}\right)_{M}^{Q}\left(t^{\alpha}\right)_{Q}^{P}\left(t^{\beta}\right)_{P}^{N}=\frac{7}{8}\left(t^{\alpha}\right)_{M}^{N}, \quad\left(t_{\alpha}\right)_{L}^{(P} K^{Q R S) L}=0 .
$$

\section{A.1 $S U(8)$ subgroup}

The maximal compact subgroup of $E_{7}$ is $S U(8)$. When $E_{7}$ is broken to $S U(8)$, the fundamental $\mathbf{5 6}$ and adjoint $\mathbf{1 3 3}$ representations break according to

$$
M=\mathbf{5 6}=\mathbf{2 8}+\overline{\mathbf{2 8}}=\left({ }_{[i j]},{ }^{[i j]}\right), \quad \alpha=\mathbf{1 3 3}=\mathbf{6 3}+\mathbf{7 0}=\left({ }_{i}{ }^{j},[i j k l]\right),
$$

where $i, j, \cdots=1, \ldots, 8$ and the $\mathbf{6 3}$ is traceless ${ }_{i}{ }^{i}=0$.

The 133 generators of $E_{7}$ break into 63 and 70 generators, respectively [57, 58]

$$
\begin{aligned}
\left(t_{i}{ }^{j}\right)_{m n}{ }^{k l} & =-\delta_{[m}^{j} \delta_{n] i}^{k l}-\frac{1}{8} \delta_{i}^{j} \delta_{m n}^{k l}=-\left(t_{i}{ }^{j}\right)^{k l}{ }_{m n} \\
\left(t_{i j k l}\right)_{m n p q} & =\frac{1}{24} \epsilon_{i j k l m n p q}, \quad\left(t_{i j k l}\right)^{m n p q}=\delta_{i j k l}^{m n p q},
\end{aligned}
$$


with Cartan-Killing metric

$$
\kappa_{m}^{n}{ }^{n}{ }^{q}=3\left(\delta_{m}^{q} \delta_{p}^{n}-\frac{1}{8} \delta_{m}^{n} \delta_{p}^{q}\right), \quad \kappa_{i j k l, m n p q}=\frac{1}{24} \epsilon_{i j k l m n p q} .
$$

The projection to the adjoint in the product $\mathbf{5 6} \times \mathbf{5 6}$ reads

$$
\begin{aligned}
(V \cdot \hat{V})_{i}{ }^{j} & =\left(V^{k j} \hat{V}_{k i}-\frac{1}{8} \delta_{i}^{j} V^{k l} \hat{V}_{k l}\right)+\left(\hat{V}^{k j} V_{k i}-\frac{1}{8} \delta_{i}^{j} \hat{V}^{k l} V_{k l}\right) \\
(V \cdot \hat{V})_{i j k l} & =-3\left(V_{[i j} \hat{V}_{k l]}+\frac{1}{4 !} \epsilon_{i j k l m n o p} V^{m n} \hat{V}^{o p}\right) .
\end{aligned}
$$

\section{A.2 $S L(2) \times O(6,6)$ subgroup}

The fundamental $\mathbf{5 6}$ representation of $E_{7}$ splits according to its $S L(2) \times O(6,6)$ subgroup as follows

$$
\begin{aligned}
\mathbf{5 6} & =(\mathbf{2}, \mathbf{1 2}) \oplus(\mathbf{1}, \mathbf{3 2}) \\
M & =(\hat{\imath} m) \oplus \alpha,
\end{aligned}
$$

where $\hat{\imath}=+,-$ is a fundamental $S L(2)$ index, $m$ an $O(6,6)$ index, and $\alpha$ is an $O(6,6)$ Majorana-Weyl spinor index.

The symplectic metric decomposes as

$$
\Omega_{M N}=\left(\begin{array}{cc}
\epsilon_{\hat{\imath} \jmath} \eta_{m n} & \\
& C_{\alpha \beta}
\end{array}\right), \quad \epsilon_{\hat{\imath} \hat{\jmath}}=\left(\begin{array}{cc}
0 & -1 \\
1 & 0
\end{array}\right), \quad \eta_{m n}=\left(\begin{array}{ll}
0 & 1 \\
1 & 0
\end{array}\right) .
$$

The different components of the projector onto the $\mathbf{1 3 3}$ representation read $[59,60]$

$$
\begin{aligned}
P_{(a d j) \hat{\imath} m \hat{\jmath} n}{ }^{\hat{k} p \hat{l} q} & =-\frac{1}{2} \epsilon_{\hat{\imath} \jmath} \epsilon^{\hat{k} \hat{l}} \delta_{m n}^{p q}+\frac{1}{12} \delta_{\hat{\imath}}^{(\hat{k}} \delta_{\hat{\jmath}}^{\hat{l})} \eta_{m n} \eta^{p q} \\
P_{(a d j) \hat{\imath} m \hat{\jmath} n}{ }^{\alpha \beta} & =-\frac{1}{8} \epsilon_{\hat{\imath} \hat{\jmath}}\left[\gamma_{m n}\right]^{\alpha \beta} \\
P_{(a d j) \alpha \beta^{\gamma \delta}} & =-\frac{1}{32}\left[\gamma_{m n}\right]_{\alpha \beta}\left[\gamma^{m n}\right]^{\gamma \delta} \\
P_{(a d j) \hat{\imath} m \alpha}{ }^{\hat{\jmath} n \beta} & =\frac{1}{24} \delta_{\hat{\imath}}^{\hat{\jmath}}\left(\left[\gamma_{m}{ }^{n}\right]_{\alpha}^{\beta}+\delta_{m}{ }^{n} \delta_{\alpha}{ }^{\beta}\right) .
\end{aligned}
$$

\section{B The Weitzenböck connection and the algebra}

Given an element $E$ of $E_{7}$

$$
E=\exp \left(\phi^{\alpha} t_{\alpha}\right)
$$

where $t_{\alpha}$ are the generators of $G$

$$
\left[t_{\alpha}, t_{\beta}\right]=f_{\alpha \beta}{ }^{\gamma} t_{\gamma}
$$

the Weitzenböck connection, defined as

$$
\Omega_{M N}^{P}=-\partial_{M}\left(E^{-1}\right)_{N}^{Q} E_{Q}^{P},
$$


is an element of the algebra of $G$. This can be easily seen by use of the identity

$$
\partial_{M} e^{X} \cdot e^{-X}=\partial_{M} X+\frac{1}{2 !}\left[X, \partial_{M} X\right]+\frac{1}{3 !}\left[X,\left[X, \partial_{M} X\right]\right]+\ldots .
$$

A quick computation shows that

$$
\Omega_{M N}^{P}=\Omega_{M}^{\alpha}\left(t_{\alpha}\right)_{N}^{P}
$$

with

$$
\Omega_{M}^{\alpha}=\partial_{M} \phi^{\alpha}-\frac{1}{2 !} f_{\sigma \beta}{ }^{\alpha} \phi^{\sigma} \partial_{M} \phi^{\beta}+\frac{1}{3 !} f_{\mu \rho}^{\sigma} f_{\beta \gamma}{ }^{\rho} \phi^{\mu} \phi^{\beta} \partial_{M} \phi^{\gamma}-\ldots
$$

\section{Representations, projectors and generalized diffeomorphisms}

In this appendix we first present the projectors onto the irreducible representations in the tensor product of the fundamental with the adjoint representation of an arbitrary simple group G, following the appendix of [41]. Related expressions and useful identities can be found in $[52,53]$. We will then show how this sheds light in the interpretation of the coefficients appearing in the structure of generalized diffeomorphisms, in terms of group theoretical quantities.

\section{C.1 Representations and projectors}

For any simple group (with the exception of $E_{8}$ ), the product of a fundamental representation $\mathbf{D}(\boldsymbol{\Lambda})$ times the adjoint decomposes in the direct sum of $\mathbf{D}(\boldsymbol{\Lambda})$ plus two other representations, $\mathbf{D}_{\mathbf{1}}$ and $\mathbf{D}_{\mathbf{2}}$, with $\operatorname{dim}\left(\mathrm{D}_{1}\right)<\operatorname{dim}\left(\mathrm{D}_{2}\right)$,

$$
\mathbf{D}(\boldsymbol{\Lambda}) \times \operatorname{Adj}(\mathbf{G}) \rightarrow \mathbf{D}(\Lambda)+\mathbf{D}_{1}+\mathbf{D}_{2}
$$

This is also true for orthogonal groups by replacing the fundamental representation by the spinor representation. Supersymmetry requires

$$
F_{M N}^{P} \in \mathbf{D}(\boldsymbol{\Lambda})+\mathbf{D}_{\mathbf{1}},
$$

and therefore it is useful to construct projectors onto these representations.

Let us call $d_{\Lambda}=\operatorname{dim}(\mathbf{D}(\boldsymbol{\Lambda})), d=\operatorname{dim}(\mathrm{G})$, and $\left\{t^{\alpha}\right\}(\alpha=1, \ldots, d)$ the generators of $\mathrm{G}$ in the $\mathbf{D}(\boldsymbol{\Lambda})$ representation. Furthermore, let $C_{\theta}, C_{\Lambda}$ be the Casimirs of the adjoint and fundamental representations, respectively. The invariant matrix $\eta^{\alpha \beta}=\operatorname{Tr}\left(t^{\alpha} t^{\beta}\right)$ is used to rise and lower the adjoint indices, and is related to the Cartan-Killing metric $\kappa^{\alpha \beta}$ by

$$
\kappa^{\alpha \beta}=\frac{d}{C_{\Lambda} d_{\Lambda}} \eta^{\alpha \beta}
$$

Using the definition of the Casimir operator, $C_{\Lambda} 1_{d_{\Lambda}}=\kappa_{\alpha \beta} t^{\alpha} t^{\beta}$, we have the following relation

$$
f_{\alpha \beta}^{\gamma} f_{\sigma}^{\alpha \beta}=-\frac{d}{d_{\Lambda}} C_{\mathrm{r}} \delta_{\sigma}^{\gamma}, \quad \text { with } \quad C_{\mathrm{r}}=\frac{C_{\theta}}{C_{\Lambda}}=\frac{d_{\Lambda}}{d} \frac{g^{\vee}}{\tilde{I}_{\Lambda}},
$$


where $g^{\vee}$ is the dual Coxeter number and $\tilde{I}_{\Lambda}$ is the Dynkin index of the fundamental representation. In the simply laced case we have additionally

$$
C_{\mathrm{r}}=\frac{d_{\Lambda}}{d}\left(\frac{d}{r}-1\right) \frac{1}{\tilde{I}_{\Lambda}}
$$

with $r$ the rank of $\mathrm{G}$.

Denote the projectors on the representations in (C.1) by $P_{(D(\Lambda))}, P_{\left(D_{1}\right)}, P_{\left(D_{2}\right)}$. These are orthonormal, i.e.

$$
P_{(X) M}{ }^{\alpha P} P_{(Y) P}{ }_{\beta}{ }_{\beta}=\delta_{X Y} P_{(X) M}^{\alpha N_{\beta}},
$$

and sum to the identity on $\mathbf{D}(\boldsymbol{\Lambda}) \times \operatorname{Adj}(\mathbf{G})$. These three projectors can be expressed in terms of three independent objects, namely:

$$
\begin{aligned}
P_{(D(\Lambda)) M}^{\alpha N_{\beta}} & =\frac{d_{\Lambda}}{d}\left(t^{\alpha} t_{\beta}\right)_{M}^{N}, \\
P_{\left(D_{1}\right) M_{\beta}^{\alpha N}} & =a_{1} \delta^{\alpha}{ }_{\beta} \delta_{M}{ }^{N}+a_{2}\left(t_{\beta} t^{\alpha}\right)_{M^{N}}+a_{3}\left(t^{\alpha} t_{\beta}\right)_{M}{ }^{N}, \\
P_{\left(D_{2}\right) M^{\alpha N}} & =\left(1-a_{1}\right) \delta^{\alpha}{ }_{\beta} \delta_{M}^{N}-a_{2}\left(t_{\beta} t^{\alpha}\right)_{M^{N}}-\left(d_{\Lambda} / d+a_{3}\right)\left(t^{\alpha} t_{\beta}\right)_{M^{N}},
\end{aligned}
$$

where

$$
\begin{aligned}
& a_{1}=\frac{\left.\left.d_{\Lambda}\left(4+\left(C_{\mathrm{r}}-4\right) d\right)\right)+d_{1}\left(\left(C_{\mathrm{r}}-2\right) d-2\right)\right)}{\left(10+d\left(C_{\mathrm{r}}-8\right)+d^{2}\left(C_{\mathrm{r}}-2\right)\right) d_{\Lambda}} \\
& a_{2}=-\frac{\left.2\left(4+\left(C_{\mathrm{r}}-4\right) d\right)\right)\left((d-1) d_{\Lambda}-2 d_{1}\right)}{\left(10+d\left(C_{\mathrm{r}}-8\right)+d^{2}\left(C_{\mathrm{r}}-2\right)\right) C_{\mathrm{r}} d} \\
& a_{3}=\frac{\left.-d_{\Lambda}\left(4+\left(C_{\mathrm{r}}-4\right) d\right)\right)\left(2+\left(C_{\mathrm{r}}-2\right) d\right)+d_{1}\left(16(d-1)-10(d-1) C_{\mathrm{r}}+C_{\mathrm{r}}^{2} d\right)}{\left(10+d\left(C_{\mathrm{r}}-8\right)+d^{2}\left(C_{\mathrm{r}}-2\right)\right) C_{\mathrm{r}} d}
\end{aligned}
$$

with $d_{1}=\operatorname{dim}\left(\mathbf{D}_{1}\right)$. Moreover, $d_{1}$ is determined to be

$$
d_{1}=\frac{d_{\Lambda}}{2}\left[d-1+\frac{\sqrt{C_{\mathrm{r}}}\left(10+d\left(C_{\mathrm{r}}-8\right)+d^{2}\left(C_{\mathrm{r}}-2\right)\right)}{\sqrt{256(d-1)+C_{\mathrm{r}}\left(100+4 d\left(5 C_{\mathrm{r}}-38\right)+\left(C_{\mathrm{r}}-2\right)^{2} d^{2}\right)}}\right] .
$$

In table 2, taken from [41], we give these coefficients for all simple Lie algebras except $\mathrm{E}_{8}$ (for which the relevant projectors have been computed in [55]).

\section{C.2 Generalized diffeomorphisms}

Let us show how these results shed light on the general structure of generalized diffeomorphisms, equation (2.8), in particular why is the proportionality coefficient between the tensor $A$ and the projector to the adjoint representation equal to 12 for $E_{7}$ (see equation (2.9)), and what it would be for other groups. Let us write the generalized diffeomorphisms (2.8) in the generic form, as in [28]

$$
\mathcal{L}_{\xi} V^{M}=\xi^{P} \partial_{P} V^{M}-\alpha P_{(a d j)}{ }^{M}{ }_{N}{ }^{P}{ }_{Q} \partial_{P} \xi^{Q} V^{N}+\frac{\omega}{2} \partial_{P} \xi^{P} V^{M},
$$

where $\alpha$ is some group-dependent constant, and the projector to the adjoint is given in (2.9). Restricted to orthogonal frames $E_{\bar{A}}$, these transformations must reproduce the embedding 


\begin{tabular}{|lccllrrr|}
\hline $\mathrm{G}$ & $g^{\vee}$ & $d_{\Lambda}$ & $\tilde{I}_{\Lambda}$ & $d_{1}$ & $a_{1}$ & $a_{2}$ & $a_{3}$ \\
\hline $\mathrm{A}_{\mathrm{r}}$ & $r+1$ & $r+1$ & $\frac{1}{2}$ & $\frac{1}{2}(r-1)(r+1)(r+2)$ & $\frac{1}{2}$ & $-\frac{1}{2}$ & $-\frac{1}{2 r}$ \\
$\mathrm{~B}_{\mathrm{r}}$ & $2 r-1$ & $2 r+1$ & 1 & $\frac{1}{3} r\left(4 r^{2}-1\right)$ & $\frac{1}{3}$ & $-\frac{2}{3}$ & 0 \\
$\mathrm{~B}_{\mathrm{r}}$ & $2 r-1$ & $2^{r}$ & $2^{r-3}$ & $2^{r+1} r$ & $\frac{2}{2 r-1}$ & $-2^{r-1} \frac{1}{2 r-1}$ & $2^{r-1} \frac{2 r-7}{4 r^{2}-1}$ \\
$\mathrm{C}_{\mathrm{r}}$ & $r+1$ & $2 r$ & $\frac{1}{2}$ & $\frac{8}{3} r\left(r^{2}-1\right)$ & $\frac{2}{3}$ & $-\frac{2}{3}$ & $-\frac{2}{1+2 r}$ \\
$\mathrm{D}_{\mathrm{r}}$ & $2 r-2$ & $2 r$ & 1 & $\frac{2}{3} r\left(2 r^{2}-3 r+1\right)$ & $\frac{1}{3}$ & $-\frac{2}{3}$ & 0 \\
$\mathrm{D}_{\mathrm{r}}$ & $2 r-2$ & $2^{r-1}$ & $2^{r-4}$ & $2^{r-1}(2 r-1)$ & $\frac{1}{r-1}$ & $-2^{r-3} \frac{1}{r-1}$ & $2^{r-3} \frac{(r-4)}{r(r-1)}$ \\
$\mathrm{G}_{2}$ & 4 & 7 & 1 & 27 & $\frac{3}{7}$ & $-\frac{6}{7}$ & $-\frac{3}{14}$ \\
$\mathrm{~F}_{4}$ & 9 & 26 & 3 & 273 & $\frac{1}{4}$ & $-\frac{3}{2}$ & $\frac{1}{4}$ \\
$\mathrm{E}_{6}$ & 12 & 27 & 3 & 351 & $\frac{1}{5}$ & $-\frac{6}{5}$ & $\frac{3}{10}$ \\
$\mathrm{E}_{7}$ & 18 & 56 & 6 & 912 & $\frac{1}{7}$ & $-\frac{12}{7}$ & $\frac{4}{7}$ \\
\hline
\end{tabular}

Table 2. Coefficients needed to construct the projectors for all simple algebras except $E_{8}$.

tensor components that are compatible with supersymmetry, i.e., the $\mathbf{D}(\boldsymbol{\Lambda})+\mathbf{D}_{\mathbf{1}}$ components, and project out the remaining representation $\mathbf{D}_{\mathbf{2}}$. Let us set $\omega=0$ for the moment, i.e., let us assume that the global symmetry group has no $\mathbb{R}^{+}$component (later we will restore $\omega$ ). In this case we find

$$
F_{M N}{ }^{P}=E^{\bar{A}}{ }_{M} E^{\bar{B}}{ }_{N} \mathcal{L}_{E_{\bar{A}}} E_{\bar{B}}{ }^{P}=\Omega_{M N}{ }^{P}-\alpha P_{(a d j)}{ }^{P}{ }_{N}{ }^{K}{ }_{L} \Omega_{K M}{ }^{L} .
$$

As we showed in (B), the Weitzenböck connection $\Omega$ takes values in the fundamental times the adjoint of the global symmetry group, so

$$
F_{M N}^{P}=F_{M}^{\alpha}\left(t_{\alpha}\right)_{N}^{P}
$$

with

$$
F_{M}^{\alpha}=Q_{M}{ }^{\alpha},{ }_{\beta}{ }_{\beta} \Omega_{N}^{\beta}, \quad Q_{M}{ }^{\alpha},{ }_{\beta}^{N}=\delta_{M}^{N} \delta_{\beta}^{\alpha}-\alpha\left(t_{\beta} t^{\alpha}\right)_{M}{ }^{N} .
$$

The coefficient $\alpha$ must then be fixed in such a way that the tensor $Q_{M}{ }^{\alpha},^{N}{ }_{\beta}$ is a linear combination of the projectors to $\mathbf{D}(\boldsymbol{\Lambda})+\mathbf{D}_{\mathbf{1}}$. We find

$$
Q_{M}^{\alpha},{ }_{\beta}=\frac{1}{a_{1}} P_{\left(D_{1}\right) M}{ }^{\alpha N_{\beta}}-\frac{a_{3}}{a_{1}} \frac{d}{d_{\Lambda}} P_{(D(\Lambda)) M}{ }_{\beta}^{\alpha N_{\beta}}
$$

provided

$$
\alpha=-\frac{a_{2}}{a_{1}}=\frac{\left.2 d_{\Lambda}\left(4+\left(C_{\mathrm{r}}-4\right) d\right)\right)\left((d-1) d_{\Lambda}-2 \Delta\right)}{\left.\left.\left[d_{\Lambda}\left(4+\left(C_{\mathrm{r}}-4\right) d\right)\right)+d_{1}\left(\left(C_{\mathrm{r}}-2\right) d-2\right)\right)\right] C_{r} d},
$$

corresponding in particular to $\alpha=12$ in $E_{7}$, as stated in equation (2.9).

Let us now see how the coefficient $\omega$ can be fixed. A possibility is to demand that the intertwining tensor (i.e. the symmetric part of the gauge group generators $F_{(M N)}{ }^{P}$ ) takes values in the algebra of the global symmetry group without the $\mathbb{R}^{+}$, as explained in $[57,58]$. Here we work out the $E_{7}$ case, but the other cases follow analogously. For a 
generic value of $\omega$, we can compute the general form of the symmetric part of the gauge group generators, which reads

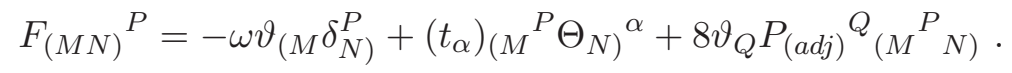

We can now use the relation (A.4), together with the fact that the $\mathbf{9 1 2}$ satisfies

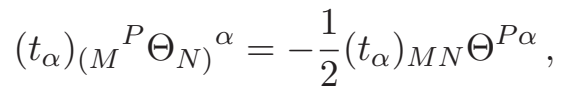

to show that (C.15) can be written as

$$
F_{(M N)}^{P}=(1-\omega) \vartheta_{(M} \delta_{N)}^{P}-\frac{1}{2}\left(\Theta^{P \alpha}-16 \vartheta_{Q}\left(t^{\alpha}\right)^{P Q}\right)\left(t_{\alpha}\right)_{M N} .
$$

Here the first term measures the failure of the intertwining tensor to take values in the algebra of $E_{7}$, and in order to cancel it we must take

$$
\omega=1 .
$$

This procedure can be repeated for any other group analogously.

In supersymmetric theories, the representation $\mathbf{D}_{\mathbf{2}}$ is projected out from the embedding tensor through a linear constraint. Given that the projectors are normalized to add to unity, the projectors to $\mathbf{D}(\boldsymbol{\Lambda})$ and $\mathbf{D}_{\mathbf{1}}$ contain information about the projector to $\mathbf{D}_{\mathbf{2}}$. This means that the coefficient $\alpha$ carries information about supersymmetry. The linear constraint is automatically engineered in the definition of the generalized Lie derivative through (C.13), which is therefore consistent with (and encodes information of) supersymmetry.

Let us conclude this section to see how the generalized diffeos of Double Field Theory and usual Riemannian geometry arise as particular examples of these generalized expressions. For DFT, with gauge group $O(d, d)$, the generators and projector to the adjoint are given by

$$
\left(t_{[M N]}\right)_{P}^{Q}=\eta_{P[M} \delta_{N]}^{Q}, \quad P_{(a d j)}{ }^{M}{ }_{N}{ }^{P} Q=\frac{1}{2}\left(\delta_{N}^{P} \delta_{Q}^{M}-\eta^{M P} \eta_{N Q}\right),
$$

and we have $\alpha=2$, so

$$
\mathcal{L}_{\xi} V^{M}=\xi^{P} \partial_{P} V^{M}-2 P_{(a d j)}{ }^{M}{ }_{N}{ }^{P}{ }_{Q} \partial_{P} \xi^{Q} V^{N}=\xi^{P} \partial_{P} V^{M}+\left(\partial^{M} \xi_{P}-\partial_{P} \xi^{M}\right) V^{P}
$$

which matches the expression of [3-6]. For usual Riemannian geometry, we have the group $G L(d)$ with generators and projector to the adjoint

$$
\left(t_{M}{ }^{N}\right)_{P}{ }^{Q}=\delta_{M}^{Q} \delta_{P}^{N}, \quad P_{(a d j)}{ }^{M}{ }_{N}^{P}{ }_{Q}=\delta_{M}^{Q} \delta_{P}^{N},
$$

and we have $\alpha=1$, so

$$
\mathcal{L}_{\xi} V^{M}=\xi^{P} \partial_{P} V^{M}-P_{(a d j)}{ }^{M}{ }_{N}^{P}{ }_{Q} \partial_{P} \xi^{Q} V^{N}=\xi^{P} \partial_{P} V^{M}-\partial_{P} \xi^{M} V^{P},
$$

which is the usual Lie derivative. 


\section{The scalar potential from extended geometry}

Here we show how the trace of the generalized Ricci tensor (5.20)

$$
2 \mathcal{R}_{\bar{A} \bar{B}}=2 W_{\bar{E}(\bar{A}} \bar{D}(W-F)_{\bar{B}) \bar{D}}{ }^{\bar{E}}+W_{\bar{D} \bar{A}}{ }^{\bar{E}} Y^{\bar{D}}{ }_{\bar{E}} \bar{F}^{\bar{G}} W_{\bar{G} \bar{B}}{ }^{\bar{G}}-8 \vartheta_{\bar{A}} \vartheta_{\bar{B}}-2 \nabla_{N} V^{N}{ }_{(\bar{A} \bar{B})},
$$

introduced in section 5 gives the scalar potential of maximal supergravity (5.26). To prove this we will use the following assumptions:

- The spin connection is torsionless (4.17)

$$
W_{A B}^{C}-12 P_{(a d j)}{ }^{C}{ }_{B}{ }^{D}{ }_{E} W_{D A}{ }^{E}=F_{A B}{ }^{C}+\vartheta_{A} \delta_{B}^{C} .
$$

From here it is also clear that it belongs to the $\mathbf{5 6} \times \mathbf{1 3 3}$ representation $W_{A B}{ }^{C}=$ $W_{A}^{\alpha}\left(t_{\alpha}\right)_{B}{ }^{C}$. This also implies that the trace is given by

$$
W_{B A}{ }^{B}=-2 \vartheta_{A} .
$$

- The spin connection is generalized metric compatible (4.18)

$$
W_{A E}{ }^{C} H^{B E}=-W_{A E}{ }^{B} H^{C E} .
$$

Under these assumptions we will show here that the generalized Ricci scalar equals the scalar potential of maximal supergravity. Let us emphasize that we will not solve equations (D.2) nor (D.4), instead we will only use them as implicit equations.

The Ricci scalar is defined as

$$
\mathcal{R}=H^{\bar{A} \bar{B}} \mathcal{R}_{\bar{A} \bar{B}}
$$

and it is convenient to split it as

$$
\mathcal{R}=\mathcal{R}_{0}-4 H^{\bar{A} \bar{B}} \vartheta_{\bar{A}} \vartheta_{\bar{B}}-H^{\bar{A} \bar{B}} \nabla_{N} V_{\bar{A} \bar{B}}^{N}
$$

where

$$
\mathcal{R}_{0}=\frac{1}{2} H^{\bar{A} \bar{B}} W_{\bar{D} \bar{A}} \bar{E}\left(2 W_{\bar{B} \bar{E}}^{\bar{D}}+Y^{\bar{D}}{ }_{\bar{E}}^{\bar{F}}{ }_{\bar{G}} W_{\bar{F} \bar{B}}^{\bar{G}}\right)-H^{\bar{A} \bar{B}} W_{\bar{E} \bar{A}} \bar{D}^{\bar{D}} F_{\bar{B} \bar{D}} \bar{E} .
$$

In $\mathcal{R}_{0}$, the $Y$ can be decomposed as in (3.6) and then using the torsionless condition (D.2) together with the trace of the spin connection (4.15) one obtains

$$
\mathcal{R}_{0}=-\frac{1}{2} H^{\bar{A} \bar{B}} W_{\bar{E} \bar{A}} \bar{D} F_{\bar{B} \bar{D}} \bar{E}+\frac{1}{2} H^{\bar{A} \bar{B}}\left(W_{\bar{D} \bar{A}} \bar{E} W_{\bar{E} \bar{B}} \bar{D}+W_{\bar{E} \bar{A}}{ }^{\bar{D}} W_{\bar{B} \bar{D}}{ }^{\bar{E}}\right),
$$

where last two terms vanish due to metric compatibility (D.4). We can now use the decomposition of the fluxes $F$ as in (2.18), (2.19) and (2.20) to obtain

$$
\mathcal{R}_{0}=-\frac{1}{2} H^{\bar{A} \bar{B}} W_{\bar{D} \bar{A}} \bar{E}\left(X_{\bar{B} \bar{E}}^{\bar{D}}+8 P_{(a d j)}{ }_{\bar{E}}^{\bar{E}}{ }_{\bar{B}} \vartheta_{\bar{F}}-\vartheta_{\bar{B}} \delta_{\bar{E}}^{\bar{D}}\right) .
$$

When the projector acts on the spin connection, one can use again the torsionless condition (D.2) and metric compatibility (D.4) to re-cast this expression in the form

$$
\mathcal{R}_{0}=-\frac{1}{2} H^{\bar{A} \bar{B}}\left(W_{\bar{D} \bar{A}}^{\bar{E}} X_{\bar{B} \bar{E}} \bar{D}+\frac{8}{3} \vartheta_{\bar{A}} \vartheta_{\bar{B}}-\frac{2}{3} F_{\bar{A} \bar{B}} \bar{F} \vartheta_{\bar{F}}\right) \text {. }
$$


Now plugging this in (D.6) we get

$$
\mathcal{R}=-\frac{1}{2} H^{\bar{A} \bar{B}}\left(W_{\bar{D} \bar{A}} \bar{E} X_{\bar{B} \bar{E}} \bar{D}+\frac{32}{3} \vartheta_{\bar{A}} \vartheta_{\bar{B}}-\frac{2}{3} F_{\bar{A} \bar{B}} \bar{F} \vartheta_{\bar{F}}\right)-H^{\bar{A} \bar{B}} \nabla_{N} V^{N}{ }_{(\bar{A} \bar{B})} .
$$

Here, the first term can be treated as follows. First, we use the fact that the last two indices of $X$ project the corresponding indices of $W$ into the adjoint

$$
X_{\bar{B} \bar{E}}^{\bar{D}} W_{\bar{D} \bar{A}}^{\bar{E}}=X_{\bar{B} \bar{E}}{ }^{\bar{D}} P_{(a d j)}{ }_{\bar{E}}^{\bar{E}} \bar{F}_{\bar{G}} W_{\bar{F} \bar{A}}^{\bar{G}},
$$

and using the torsionless condition (D.2) one obtains

$$
-\frac{1}{2} H^{\bar{A} \bar{B}} W_{\bar{D} \bar{A}}{ }^{\bar{E}} X_{\bar{B} \bar{E}} \bar{D}=-\frac{1}{4} H^{\bar{A} \bar{B}}\left(X_{\bar{B} \bar{F}}^{\bar{G}} W_{\bar{A} \bar{G}} \bar{F}-X_{\bar{B} \bar{F}}{ }^{\bar{G}} F_{\bar{A} \bar{G}}{ }^{\bar{F}}\right) .
$$

Finally, the first term here can be massaged by explicitly extracting a projector to the $\mathbf{9 1 2}$ from $X$

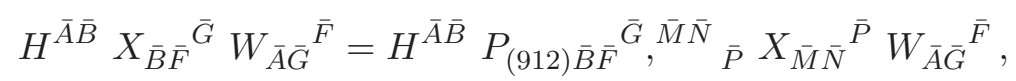

and exploiting the fact that the projector is invariant under rotations with the generalized metric

$$
P_{(912) \bar{A} \bar{B}} \bar{C},{ }^{\bar{M}} \bar{N}{ }_{\bar{P}} H^{\bar{A}}{ }_{\bar{A}^{\prime}} H^{\bar{B}} \bar{B}^{\prime} H_{\bar{C}} \bar{C}^{\prime} H_{\bar{M}} \bar{M}^{\prime} H_{\bar{N}} \bar{N}^{\prime} H^{\bar{P}} \bar{P}^{\prime}=P_{(912) \bar{A}^{\prime} \bar{B}^{\prime}} \bar{C}^{\prime}, \bar{M}^{\prime} \bar{N}^{\prime} \bar{P}^{\prime},
$$

after some algebra one obtains the final result

$$
\begin{aligned}
\frac{1}{4} \mathcal{R}= & \frac{1}{672}\left[H^{\bar{A} \bar{D}} \bar{H}^{\bar{B} \bar{E}} H_{\bar{C} \bar{F}} X_{\bar{A} \bar{B}} \bar{C}^{\bar{C}} X_{\bar{D} \bar{E}} \bar{F}+7 H^{\bar{A} \bar{B}} X_{\bar{A} \bar{C}} \bar{D} F_{\bar{B} \bar{D}} \bar{C}\right] \\
& -\frac{4}{3} H^{\bar{A} \bar{B}} \vartheta_{\bar{A}} \vartheta_{\bar{B}}+\frac{1}{12} H^{\bar{A} \bar{B}} F_{\bar{A} \bar{B}} \bar{F} \vartheta_{\bar{F}}-\frac{1}{4} H^{\bar{A} \bar{B}} \nabla_{N} V^{N}{ }_{(\bar{A} \bar{B})}
\end{aligned}
$$

Being expressed purely in terms of fluxes, we see that the undetermined pieces of the spin connection dropped out. Remarkably, this takes the exact same form as the scalar potential of gauged maximal supergravity [54] if we identify the generalized metric with the moduli space metric $\mathcal{M}$ and take the gaugings in the $\mathbf{5 6}$ to vanish

$$
\frac{1}{4} \mathcal{R}=\frac{1}{672}\left[H^{\bar{A} \bar{D}} \bar{H}^{\bar{B} \bar{E}} H_{\bar{C} \bar{F}} X_{\bar{A} \bar{B}}^{\bar{C}} X_{\bar{D} \bar{E}}^{\bar{F}}+7 H^{\bar{A} \bar{B}} X_{\bar{A} \bar{C}}^{\bar{D}} X_{\bar{B} \bar{D}} \bar{C}\right] .
$$

Note also that this is true for any torsionless and metric-compatible connection, and the concrete expression of the determined part does not need to be known. In fact, we never needed to solve for the spin connection, but only used the equation that defines it implicitly.

Finally, let us mention that the factor 7 in the scalar potential comes form a projection of the spin connection into the space of fluxes (4.16). It is known that it is fixed by supersymmetry [54], so one can wonder where does supersymmetry arise in all this analysis. This factor is actually $1 / a_{1}$ in the language of appendix $\mathrm{C}$, and it is set by supersymmetry in an indirect way. As we explained in appendix $\mathrm{C}$, the projectors to the irreducible reps of the direct product of the fundamental and the adjoint are normalized to add up to unity. Since supersymmetry projects out some reps through a linear constraint, the remaining are normalized in such a way that they capture information about supersymmetry and this is exactly how this coefficient is obtained here. 
Open Access. This article is distributed under the terms of the Creative Commons Attribution License which permits any use, distribution and reproduction in any medium, provided the original author(s) and source are credited.

\section{References}

[1] N. Hitchin, Generalized Calabi-Yau manifolds, Quart. J. Math. Oxford Ser. 54 (2003) 281 [math/0209099] [INSPIRE].

[2] M. Gualtieri, Generalized complex geometry, math/0401221 [INSPIRE].

[3] C. Hull and B. Zwiebach, Double Field Theory, JHEP 09 (2009) 099 [arXiv:0904.4664] [INSPIRE].

[4] C. Hull and B. Zwiebach, The Gauge algebra of double field theory and Courant brackets, JHEP 09 (2009) 090 [arXiv:0908.1792] [INSPIRE].

[5] O. Hohm, C. Hull and B. Zwiebach, Background independent action for double field theory, JHEP 07 (2010) 016 [arXiv: 1003.5027] [INSPIRE].

[6] O. Hohm, C. Hull and B. Zwiebach, Generalized metric formulation of double field theory, JHEP 08 (2010) 008 [arXiv: 1006.4823] [INSPIRE].

[7] C. Hull and R. Reid-Edwards, Gauge symmetry, T-duality and doubled geometry, JHEP 08 (2008) 043 [arXiv: 0711.4818] [INSPIRE].

[8] C. Hull and R. Reid-Edwards, Flux compactifications of M-theory on twisted Tori, JHEP 10 (2006) 086 [hep-th/0603094] [INSPIRE].

[9] C.M. Hull, Doubled Geometry and T-Folds, JHEP 07 (2007) 080 [hep-th/0605149] [INSPIRE].

[10] A. Dabholkar and C. Hull, Generalised T-duality and non-geometric backgrounds, JHEP 05 (2006) 009 [hep-th/0512005] [INSPIRE].

[11] C. Hull, A Geometry for non-geometric string backgrounds, JHEP 10 (2005) 065 [hep-th/0406102] [INSPIRE].

[12] O. Hohm and S.K. Kwak, Massive Type II in Double Field Theory, JHEP 11 (2011) 086 [arXiv: 1108.4937] [INSPIRE].

[13] M. Graña and D. Marques, Gauged Double Field Theory, JHEP 04 (2012) 020 [arXiv: 1201.2924] [INSPIRE].

[14] G. Dall'Agata, N. Prezas, H. Samtleben and M. Trigiante, Gauged Supergravities from Twisted Doubled Tori and Non-Geometric String Backgrounds, Nucl. Phys. B 799 (2008) 80 [arXiv:0712.1026] [INSPIRE].

[15] G. Aldazabal, E. Andres, P.G. Camara and M. Graña, U-dual fluxes and Generalized Geometry, JHEP 11 (2010) 083 [arXiv: 1007.5509] [INSPIRE].

[16] C. Hull, Generalised Geometry for M-theory, JHEP 07 (2007) 079 [hep-th/0701203] [INSPIRE].

[17] P.P. Pacheco and D. Waldram, M-theory, exceptional generalised geometry and superpotentials, JHEP 09 (2008) 123 [arXiv:0804.1362] [INSPIRE]. 
[18] D.S. Berman, H. Godazgar, M. Godazgar and M.J. Perry, The Local symmetries of M-theory and their formulation in generalised geometry, JHEP 01 (2012) 012 [arXiv:1110.3930] [INSPIRE].

[19] D.S. Berman, H. Godazgar, M.J. Perry and P. West, Duality Invariant Actions and Generalised Geometry, JHEP 02 (2012) 108 [arXiv:1111.0459] [INSPIRE].

[20] D.S. Berman and M.J. Perry, Generalized Geometry and M-theory, JHEP 06 (2011) 074 [arXiv: 1008.1763] [INSPIRE].

[21] D.C. Thompson, Duality Invariance: from M-theory to Double Field Theory, JHEP 08 (2011) 125 [arXiv: 1106.4036] [INSPIRE].

[22] A. Coimbra, C. Strickland-Constable and D. Waldram, $E_{d(d)} \times \mathbb{R}^{+}$Generalised Geometry, Connections and $M$-theory, arXiv:1112.3989 [INSPIRE].

[23] A. Coimbra, C. Strickland-Constable and D. Waldram, Supergravity as Generalised Geometry II: $E_{d(d)} \times \mathbb{R}^{+}$and M-theory, arXiv:1212.1586 [INSPIRE].

[24] O. Hohm, S.K. Kwak and B. Zwiebach, Unification of Type II Strings and T-duality, Phys. Rev. Lett. 107 (2011) 171603 [arXiv:1106.5452] [INSPIRE].

[25] O. Hohm, S.K. Kwak and B. Zwiebach, Double Field Theory of Type II Strings, JHEP 09 (2011) 013 [arXiv:1107.0008] [INSPIRE].

[26] A. Coimbra, C. Strickland-Constable and D. Waldram, Supergravity as Generalised Geometry I: Type II Theories, JHEP 11 (2011) 091 [arXiv:1107.1733] [INSPIRE].

[27] D.S. Berman, E.T. Musaev, D.C. Thompson and D.C. Thompson, Duality Invariant M-theory: Gauged supergravities and Scherk-Schwarz reductions, JHEP 10 (2012) 174 [arXiv: 1208.0020$]$ [INSPIRE].

[28] D.S. Berman, M. Cederwall, A. Kleinschmidt and D.C. Thompson, The gauge structure of generalised diffeomorphisms, JHEP 01 (2013) 064 [arXiv:1208.5884] [INSPIRE].

[29] W. Siegel, Superspace duality in low-energy superstrings, Phys. Rev. D 48 (1993) 2826 [hep-th/9305073] [INSPIRE].

[30] W. Siegel, Two vierbein formalism for string inspired axionic gravity, Phys. Rev. D 47 (1993) 5453 [hep-th/9302036] [INSPIRE].

[31] S. Guttenberg, Brackets, $\sigma$-models and Integrability of Generalized Complex Structures, JHEP 06 (2007) 004 [hep-th/0609015] [INSPIRE].

[32] A.A. Tseytlin, Duality symmetric closed string theory and interacting chiral scalars, Nucl. Phys. B 350 (1991) 395 [InSPIRE].

[33] I. Jeon, K. Lee and J.-H. Park, Differential geometry with a projection: application to double field theory, JHEP 04 (2011) 014 [arXiv: 1011.1324] [INSPIRE].

[34] I. Jeon, K. Lee and J.-H. Park, Stringy differential geometry, beyond Riemann, Phys. Rev. D 84 (2011) 044022 [arXiv: 1105.6294] [inSPIRE].

[35] O. Hohm and S.K. Kwak, Frame-like Geometry of Double Field Theory, J. Phys. A 44 (2011) 085404 [arXiv: 1011.4101] [INSPIRE].

[36] O. Hohm and B. Zwiebach, On the Riemann Tensor in Double Field Theory, JHEP 05 (2012) 126 [arXiv:1112.5296] [INSPIRE]. 
[37] O. Hohm and B. Zwiebach, Towards an invariant geometry of double field theory, arXiv:1212.1736 [INSPIRE].

[38] G. Aldazabal, W. Baron, D. Marques and C. Núñez, The effective action of Double Field Theory, JHEP 11 (2011) 052 [Erratum ibid. 1111 (2011) 109] [arXiv:1109. 0290] [INSPIRE].

[39] D. Geissbuhler, Double Field Theory and $N=4$ Gauged Supergravity, JHEP 11 (2011) 116 [arXiv: 1109.4280] [INSPIRE].

[40] O. Hohm and S.K. Kwak, Double Field Theory Formulation of Heterotic Strings, JHEP 06 (2011) 096 [arXiv:1103.2136] [INSPIRE].

[41] B. de Wit, H. Samtleben and M. Trigiante, On Lagrangians and gaugings of maximal supergravities, Nucl. Phys. B 655 (2003) 93 [hep-th/0212239] [INSPIRE].

[42] E.T. Musaev, Gauged supergravities in 5 and 6 dimensions from generalised Scherk-Schwarz reductions, arXiv: 1301.0467 [INSPIRE].

[43] P. West, Generalised geometry, eleven dimensions and E11, JHEP 02 (2012) 018 [arXiv:1111.1642] [INSPIRE].

[44] P. West, Generalised space-time and duality, Phys. Lett. B 693 (2010) 373 [arXiv: 1006.0893] [INSPIRE].

[45] F. Riccioni and P.C. West, $E_{11}$-extended spacetime and gauged supergravities, JHEP 02 (2008) 039 [arXiv:0712.1795] [INSPIRE].

[46] E. Malek, U-duality in three and four dimensions, arXiv:1205.6403 [INSPIRE].

[47] D. Andriot, O. Hohm, M. Larfors, D. Lüst and P. Patalong, A geometric action for non-geometric fluxes, Phys. Rev. Lett. 108 (2012) 261602 [arXiv:1202.3060] [INSPIRE].

[48] D. Andriot, O. Hohm, M. Larfors, D. Lüst and P. Patalong, Non-Geometric Fluxes in Supergravity and Double Field Theory, Fortsch. Phys. 60 (2012) 1150 [arXiv:1204.1979] [INSPIRE].

[49] R. Blumenhagen, A. Deser, E. Plauschinn and F. Rennecke, Non-geometric strings, symplectic gravity and differential geometry of Lie algebroids, JHEP 02 (2013) 122 [arXiv: 1211.0030] [INSPIRE].

[50] J.-H. Park and Y. Suh, U-geometry: SL(5), JHEP 04 (2013) 147 [arXiv:1302.1652] [INSPIRE].

[51] A. Chatzistavrakidis and L. Jonke, Matrix theory origins of non-geometric fluxes, JHEP 02 (2013) 040 [arXiv: 1207.6412] [INSPIRE].

[52] F. Riccioni and P.C. West, The $E_{11}$ origin of all maximal supergravities, JHEP 07 (2007) 063 [arXiv:0705.0752] [INSPIRE].

[53] F. Riccioni, D. Steele and P. West, The $E_{11}$ origin of all maximal supergravities: the Hierarchy of field-strengths, JHEP 09 (2009) 095 [arXiv:0906.1177] [INSPIRE].

[54] B. de Wit, H. Samtleben and M. Trigiante, The Maximal D=4 supergravities, JHEP 06 (2007) 049 [arXiv:0705.2101] [INSPIRE].

[55] K. Koepsell, H. Nicolai and H. Samtleben, On the Yangian $\left[Y\left(E_{8}\right)\right]$ quantum symmetry of maximal supergravity in two-dimensions, JHEP 04 (1999) 023 [hep-th/9903111] [INSPIRE].

[56] G. Dibitetto, J. Fernandez-Melgarejo, D. Marques and D. Roest, Duality orbits of non-geometric fluxes, Fortsch. Phys. 60 (2012) 1123 [arXiv:1203.6562] [INSPIRE]. 
[57] A. Le Diffon and H. Samtleben, Supergravities without an Action: Gauging the Trombone, Nucl. Phys. B 811 (2009) 1 [arXiv:0809.5180] [InSPIRE].

[58] A. Le Diffon, H. Samtleben and M. Trigiante, $N=8$ Supergravity with Local Scaling Symmetry, JHEP 04 (2011) 079 [arXiv:1103.2785] [INSPIRE].

[59] G. Dibitetto, A. Guarino and D. Roest, How to halve maximal supergravity, JHEP 06 (2011) 030 [arXiv:1104.3587] [INSPIRE].

[60] G. Dibitetto, A. Guarino and D. Roest, Exceptional Flux Compactifications, JHEP 05 (2012) 056 [arXiv: 1202.0770] [INSPIRE].

[61] M. Graña, J. Louis, A. Sim and D. Waldram, $E_{7(7)}$ formulation of $N=2$ backgrounds, JHEP 07 (2009) 104 [arXiv:0904.2333] [INSPIRE].

[62] G. Dall'Agata, G. Inverso and M. Trigiante, Evidence for a family of $\mathrm{SO}(8)$ gauged supergravity theories, Phys. Rev. Lett. 109 (2012) 201301 [arXiv:1209.0760] [INSPIRE].

[63] M. Cederwall, J. Edlund and A. Karlsson, Exceptional geometry and tensor fields, arXiv:1302.6736 [INSPIRE]. 